ARTICLE

DOI: $10.1038 / \mathrm{s} 41467-017-02149-0$

\title{
Balancing mcr-1 expression and bacterial survival is a delicate equilibrium between essential cellular defence mechanisms
}

Qiue Yang ${ }^{1}$, Mei Li ${ }^{1}$, Owen B. Spiller ${ }^{1}$, Diego O. Andrey (1) ${ }^{1,2}$, Philip Hinchliffe ${ }^{3}$, Hui Li ${ }^{4}$, Craig MacLean (1) ${ }^{5}$, Pannika Niumsup ${ }^{6}$, Lydia Powell ${ }^{7}$, Manon Pritchard ${ }^{7}$, Andrei Papkou ${ }^{5}$, Yingbo Shen ${ }^{8}$, Edward Portal ${ }^{1}$, Kirsty Sands ${ }^{1}$, James Spencer ${ }^{3}$, Uttapoln Tansawai ${ }^{6}$, David Thomas ${ }^{7}$, Shaolin Wang ${ }^{8}$, Yang Wang ${ }^{8}$, Jianzhong Shen $^{8} \&$ Timothy Walsh ${ }^{1}$

MCR-1 is a lipid A modifying enzyme that confers resistance to the antibiotic colistin. Here, we analyse the impact of MCR-1 expression on E. coli morphology, fitness, competitiveness, immune stimulation and virulence. Increased expression of $\mathrm{mcr}-1$ results in decreased growth rate, cell viability, competitive ability and significant degradation in cell membrane and cytoplasmic structures, compared to expression of catalytically inactive MCR-1 (E246A) or MCR-1 soluble component. Lipopolysaccharide (LPS) extracted from mcr-1 strains induces lower production of IL-6 and TNF, when compared to control LPS. Compared to their parent strains, high-level colistin resistance mutants (HLCRMs) show reduced fitness (relative fitness is 0.41-0.78) and highly attenuated virulence in a Galleria mellonella infection model. Furthermore, HLCRMs are more susceptible to most antibiotics than their respective parent strains. Our results show that the bacterium is challenged to find a delicate equilibrium between expression of MCR-1-mediated colistin resistance and minimalizing toxicity and thus ensuring cell survival.

\footnotetext{
${ }^{1}$ Department of Medical Microbiology and Infectious Disease, Division of Infection and Immunity, Cardiff University, Cardiff CF14 4XN, UK. ${ }^{2}$ Service of Infectious Diseases, Geneva University Hospitals and Faculty of Medicine, 1211 Geneva, Switzerland. ${ }^{3}$ School of Cellular and Molecular Medicine, University of Bristol, Bristol BS8 1TD, UK. ${ }^{4}$ Beijing Key Laboratory of Diagnostic and Traceability Technologies for Food Poisoning, Beijing Center for Disease Prevention and Control, Beijing 100013, China. ${ }^{5}$ Department of Zoology, University of Oxford, South Parks Road, Oxford OX1 3PS, UK. ${ }^{6}$ Department of Microbiology and Parasitology, Faculty of Medical Science, Naresuan University, Phitsanulok 65000, Thailand. ${ }^{7}$ Advanced Therapies Group, Oral and Biomedical Sciences, School of Dentistry, College of Biomedical and Life Sciences, Cardiff University, Heath Park, Cardiff CF14 4XN, UK. ${ }^{8}$ Beijing Advanced Innovation Centre for Food Nutrition and Human Health, College of Veterinary Medicine, China Agricultural University, Beijing 100193, China. Correspondence and requests for materials should be addressed to Q.Y. (email: YangQe@cardiff.ac.uk) or to T.W. (email: WalshTR@cardiff.ac.uk)
} 
T he global concern over colistin resistance has profound implications as to how we successfully manage and treat serious Gram-negative infections, particularly those caused by Enterobacteriaceae ${ }^{1,2}$. Until recently, our understanding of colistin resistance was limited to chromosomal changes such as the $p m r A / p m r B$ activation of arnBCADTEF and $p m r E$, that collectively modifies lipopolysaccharide (LPS), the component of the Gramnegative outer membrane by the addition of 4-amino-4-deoxy-Larabinose ${ }^{3}$. However, the advent and subsequent reporting of plasmid-mediated colistin resistance, $m c r-1$, in both animal and human health, has invoked commentaries announcing a return to the pre-antibiotic era. Since our lab described $m c r-1$ in November 2015 , it has been reported in over 40 countries. Regrettably, as the main bacterial host of $m c r-1$ is Escherichia coli, there are also significant public health ${ }^{4}$ and environmental issues as MCR-1positive E. coli (MCRPEC) has been reported from migratory birds $^{4,5}$, flies ${ }^{4}, \operatorname{dogs}^{4}$, imported reptiles ${ }^{6}$, rivers ${ }^{7}$ and inevitably public water facilities ${ }^{8}$. The widespread reporting of $m c r-1$ since the first sequence was published is unprecedented above any previous resistance mechanism ${ }^{9,10}$. This is, in part, also due to increased surveillance; nonetheless, its rapid global dissemination only further increases the critical need for better global biosafety containment and novel drug development.

The clinical impact of $m c r-1$ is still unknown. Of the few clinical studies reporting MCRPEC from infections, most lack assessment of risk factors to determine whether MCRPEC have a different pathogenic profile to non-MCRPEC ${ }^{11-14}$. In China where MCRPEC is disseminated throughout the Chinese food industry and environment, it has been shown to colonise healthy volunteers and patients ${ }^{15}$. Whether the expression of $m c r-1$ gene modulates pathogenicity in E. coli remains to be explored.

Those few studies that have examined the association of MCRPEC with virulent factors and pathogenicity have shown little or no difference ${ }^{16}$. However, these studies involved only a few isolates and applied different methods, so a direct correlation remains unclear. Notwithstanding, $m c r-1$ plasmids have been reported in variety of different MLST clades only some of which fall into the B2 group of human pathogens ${ }^{10,16}$. Most noticeable, is that $m c r-1$ is now starting to emerge in ST131, a virulent strain associated with a variety of clinical infections ${ }^{10,17}$. However, mcr-1 encodes an enzyme that catalyses the transfer of phosphoethanolamine onto a phosphate of the $\mathrm{N}$-acetylglucosamine head group of lipid A in the bacterial outer membrane (Fig. 1) 18,19 , which may modify the structure of lipid A and alter its ability to induce the innate immune response and modify the clinical pathogenicity of bacterial infections. Furthermore, colistin MICs displayed by MCRPEC are moderate (usually 2-8 $\mathrm{mg} \mathrm{l}^{-1}$ ) when compared to the level of colistin resistance (usually 8-256 $\mathrm{mg} \mathrm{l}^{-1}$ ) mediated by, for example, increased expression of $p m r A / p m r B$, inferring that the expression of $m c r-1$ is tightly controlled $^{3,9,10,16}$. This, in part, is supported by recent studies on plasmid copy number where $m c r-1$ were only found on plasmids of a relatively low copy number ${ }^{20,21}$.

Here, we investigate the impact of $m c r-1$ expression on E. coli survival, fitness and virulence. Furthermore, we analyse the viability, virulence and high-level colistin resistance stability of MCRPEC high-level colistin-resistant mutants.

\section{Results}

Generic background and plasmid copy number. The wild-type MCRPEC isolates (PN16, PN21, PN23, PN24, PN25, PN42 and PN43) chosen for this study were selected from a collection from Phitsanulok, Thailand (Supplementary Table 1) and in keeping with the known epidemiology of MCRPEC 4 . Isolates were chosen from a variety of sources: chicken meat (PN16), chicken faeces (PN21), duck faeces (PN23, PN24 and PN25) and human faeces (PN42 and PN43) (Supplementary Table 1). Isolates PN16 and PN21, possessed $m c r-1$ plasmids with an Incl2 backbone and PN23, PN24, PN25 and PN42 possessed IncX4 mor-1 plasmids. Unusually, mcr-1 was carried on the chromosome of isolate PN43 (Supplementary Fig. 1). The genomes of the strains were sequenced and varied considerably in the number and variety of antibiotic resistance genes. For example, apart from $m c r-1$, PN23 possessed one additional gene, tet $B$, where as PN42 carried numerous antibiotic resistance genes (Supplementary Table 1). The genetic maps showing the immediate context of $\mathrm{mcr}-1$ in the wild-type isolates are shown in Supplementary Fig. 2. The MLSTs for each MCRPEC were grouped as follows: ST2040 (PN16), new variant of ST24 (PN21), ST1211 (PN23), ST3631 (PN24), ST101 (PN25), ST744 (PN42) and ST410 (PN43), confirming that the

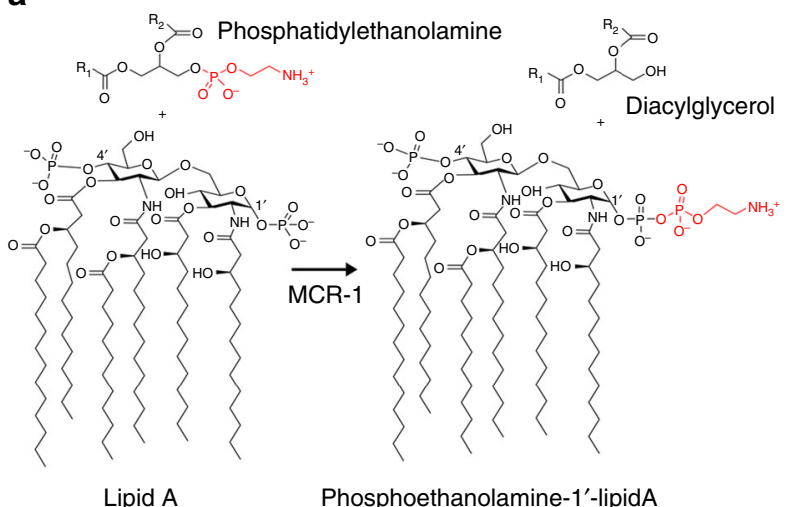

b

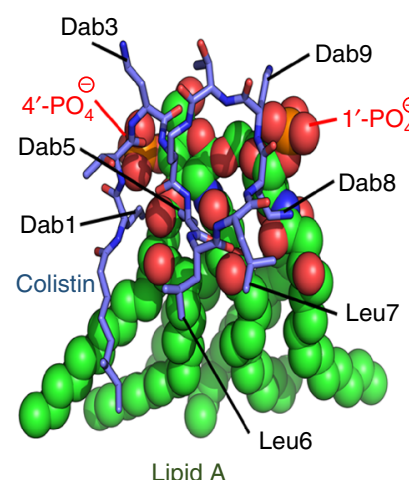

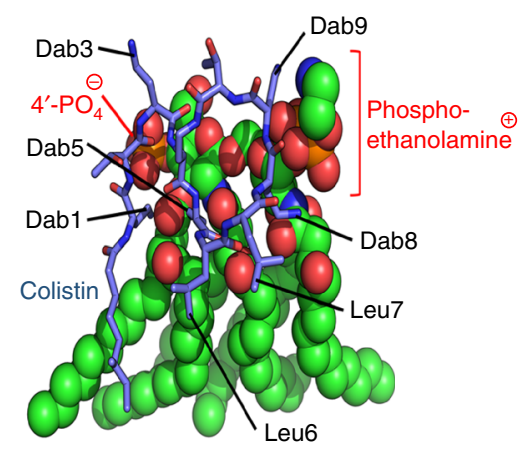

Phosphoethanolamine-1'-lipid A

Fig. 1 Molecular model of colistin binding to lipid A. a Schematic of phosphoethanolamine transfer to the 1-PO 4 group of hexa-acylated lipid A as catalysed by MCR-1. b Models of colistin (blue sticks) binding to lipid A (left) or phosphoethanolamine-lipid A (right) (spheres coloured green, red, blue and orange for $\mathrm{C}, \mathrm{O}, \mathrm{N}$ and $\mathrm{P}$ atoms, respectively). The model is based on the NMR and docking studies of polymixin B binding to lipid A with lipid A coordinates from PDB $3 \mathrm{fxi}^{22}$ and colistin coordinates adapted from the NMR structure of polymixin B bound to lipid $A^{23}$. The positively charged Dab colistin residues closely interact with the negatively-charged $1^{\prime}$ and $4^{\prime}$ phosphate groups of lipid $A$, reducing the net-negative charge of lipid $A$. The hydrophobic leucine residues and tail of colistin A interact with the fatty acid tails of lipid A, allowing colistin A to insert into, and disrupt, the bacterial outer membrane. $\mathbf{b}$ (right), model of colistin binding to phosphoethanolamine-lipid $\mathrm{A}$ indicates addition of positively charged phosphoethanolamine onto the $1^{\prime}$ - $\mathrm{PO}_{4}$ of lipid $\mathrm{A}$ likely interferes with the interaction of positively charged Dab8 and Dab9 side chains with the phosphate group, preventing colistin binding to the outer membrane of Gram-negative bacteria. Figure created using Pymol (https://www.pymol.org/) 
a

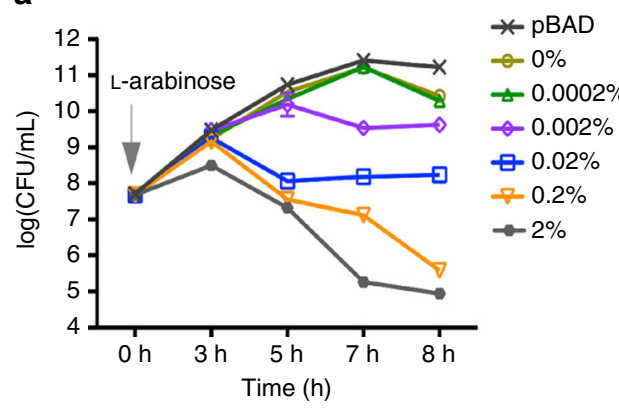

b

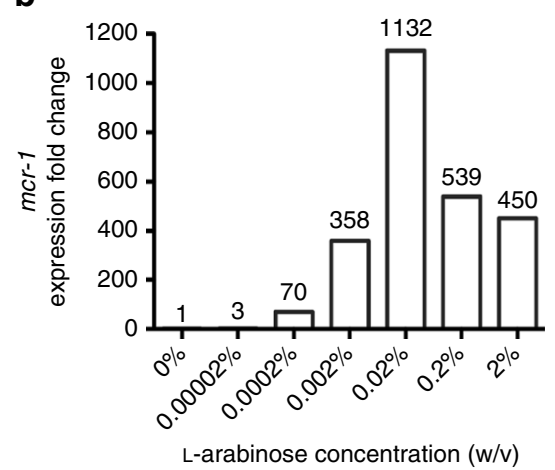

C

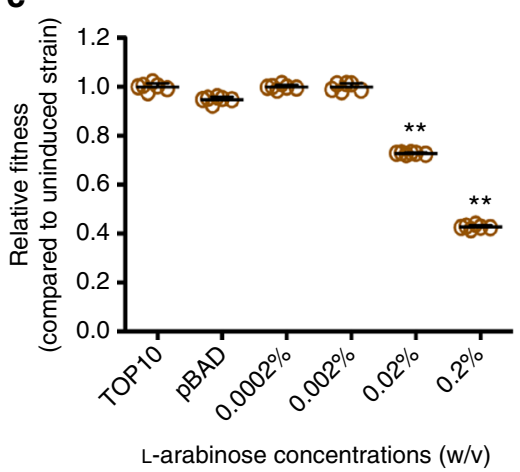

Fig. 2 Effects of $m c r-1$ overexpression on bacterial growth and fitness in vitro. a Overproducing mcr-1 causes variable effects on growth rate depending on the concentrations of L-arabinose $(n=3)$. $\mathbf{b}$ The expression levels of $m c r-1$ gene induced by increasing concentrations of arabinose were measured by qRTPCR $(n=2)$. c Relative fitness of $m c r-1$ overexpressing strain mcr-1/pBAD competing control strain pHT315 under increasing concentrations of L-arabinose $(0.0002 \%$ vs $0 \%, 0.002 \%$ vs $0 \%, 0.02 \%$ vs $0 \%, 0.2 \%$ vs $0 \%)$. Error bars represent the SD $(n=6)$. The differences in fitness were tested using nonparametric Mann-Whitney test, ${ }^{\star \star}$ indicates the $p$ values is $<0.05$. The average relative fitness and $p$ values are listed in Supplementary Table 6

MCRPEC isolates used in this study are not clonal (Supplementary Table 1). MRCPEC possessing some of these MLST groups have been reported previously ${ }^{10,16}$. The copy number of $\mathrm{mcr}-1$ was assessed by qPCR (quantitative PCR) using $16 \mathrm{~S}$ ribosomal RNA as a standard reference copy number. All isolates possessed $m c r-1$ at a copy number ranging between 1 (chromosomal copy of isolate PN43) and 5.6 in keeping with other recent studies ${ }^{20,21}$ (Supplementary Table 1).

Effects of $\boldsymbol{m c r}-1$ overexpression on bacterial growth and fitness. The $m c r-1$ coding region obtained from pHNSHP45 was constructed into plasmid pBAD to generate strain E. coli TOP10 $\mathrm{mcr}$ 1/pBAD (mcr-1/pBAD) (Supplementary Table 2, see Methods). To determine whether $m c r-1$ affects bacterial growth rate and fitness, we examined growth curves, quantitative real-time PCR (qRT-PCR) and competition assays for E. coli TOP10 carrying the mcr-1-pBAD plasmid construct. The expression level of $m c r-1$ was induced by increasing concentrations of $\mathrm{L}$-arabinose and measured by qRT-PCR (Fig. 2b). Maximal $m c r-1$ induction was observed at $0.02 \%$ L-arabinose, where $m c r-1$ expression was approximately twofold and threefold more than the arabinose concentrations of $0.002 \%$ and $0.2 \%$, respectively. As shown in Fig. $2 \mathrm{a}$, after $8 \mathrm{~h}$, the growth rates of $E$. coli strains induced by $0.2 \%$ and $0.02 \%(\mathrm{w} / \mathrm{v})$ of L-arabinose showed approximately a 3$\log _{10}$ unit decrease when compared to E.coli TOP10 (mcr-1/ pBAD) without L-arabinose. As the initial inoculum was $\sim 7.5$ $\log _{10}$ (c.f.u. per $\mathrm{ml}$ ), and this decreased to $\sim 5 \log _{10}$, it also suggests overexpression of $m c r-1$ decreases cell populations. In vitro competition experiments were also performed to determine the effect of $m c r-1$ expression on the relative fitness of $E$. coli $(\mathrm{mcr}-1 /$ pBAD) with different $m c r-1$ levels (Methods). Results are shown in Fig. $2 \mathrm{c}$ and indicate that increased $m c r-1$ expression levels were associated with a significant fitness burden in vitro. For example, inducing high levels of $m c r-1$ expression with $0.2 \%$ L-arabinose remarkably decreases relative fitness (average relative fitness 0.43 , $p=0.0022$, using non-parametric Mann-Whitney test) by $>50 \%$ relative to uninduced controls (Fig. 2c and Supplementary Table 6). To eliminate these observations being singularly due to the overexpression of a random protein, our negative control, $b l a_{\text {TEM-1b }}$, when overexpressed showed very similar growth curves to the uninduced E. coli cells (Supplementary Fig. 4C).

Overexpression of $\mathbf{m c r}-1$ affects cellular morphology. As $m c r-1$ encodes the transfer of phosphoethanolamine onto a phosphate of the $\mathrm{N}$-acetylglucosamine head group of lipid $\mathrm{A}$ in the bacterial outer membrane ${ }^{9}$. We employed transmission electron microscopy (TEM) and study the cellular morphology of E. coli (TOP10) under induced $(0.2 \%(\mathrm{w} / \mathrm{v})$ L-arabinose) and non-induced conditions (Fig. 3). Both control strains, E. coli TOP10 with pBAD minus $m c r-$ 1, and E. coli TOP10 ( $m c r-1 / \mathrm{pBAD})$ without L-arabinose induction, showed normal cellular characteristics with a multi-layered cell surface consisting of a distinct, structured outer membrane, a peptidoglycan layer in the periplasmic space, a normal cytoplasmic membrane and typically granular cytoplasm (Fig. 3a and Fig. 3b). Significant morphological changes were however, observed in $E$. coli TOP10 ( $m c r-1 / \mathrm{pBAD})$ treated with $0.2 \% \mathrm{~L}$-arabinose for $8 \mathrm{~h}$ (Fig. 3c). In particular, with the outer cell membrane cell envelope (Fig. 3f). The outer membrane region exhibited altered structural integrity, varying markedly in thickness and density, and it could not be differentiated from the cell wall or the cytoplasmic membrane (Fig. 3d-f). TEM analysis on E. coli where $m c r-1$ is overexpressed shows other gross cellular changes including complete loss of the 'bacilli' morphology and absence of electron-dense material appeared as empty 'ghost' cells (Fig. 3c). By comparison, E. coli TOP10 with pBAD minus $m c r-1$, and E. coli TOP10 ( $m c r-1 /$ pBAD) without $\mathrm{L}$-arabinose induction possess homogeneous electron densities in the cytoplasm and exhibited unaltered multilayers of cell membrane (Fig. 3a, b) confirming that it is the highlevel expression of $m c r-1$ that has induced these gross morphological changes. In order to rule out effects caused by high concentration of L-arabinose, we examined E. coli TOP10 with pBAD alone (minus $m c r-1$ ) with $0.2 \% \mathrm{~L}$-arabinose induction (Supplementary Fig. 3). Induction resulted in an intact cell wall with a well-defined inner and outer membrane, and a highly homogeneous electron density in cytoplasm were observed (Supplementary Fig. 3), further supporting that cell membrane impairment is due to the expression of $m c r-1$ gene only.

Effects of $m c r-1$ overexpression on bacterial survival. As the growth curves from Fig. 2a suggest that overexpression of $\mathrm{mcr}-1$ reduces cell counts, and given its profound effect on the $E$. coli cell structure and morphology, we investigated the effects of $m c r-$ 1 on cellular viability and biofilm assembly, using LIVE/DEAD ${ }^{\circledR}$ staining with confocal laser scanning microscopy (CLSM) imaging and COMSTAT analysis in E. coli TOP10 ( $m c r-1 / \mathrm{pBAD})$ biofilms with/without $0.2 \%$ L-arabinose induction (Fig. 4). The observed marked reduction of viability in bacterial cell overexpressing $m c r-1$ (Fig. 4), was contrast with the minimal reduction in bacterial viability observed in E. coli TOP10 with pBAD minus $m c r-1$, and $E$. coli TOP10 ( $m c r-1 /$ pBAD minus L-arabinose 
a

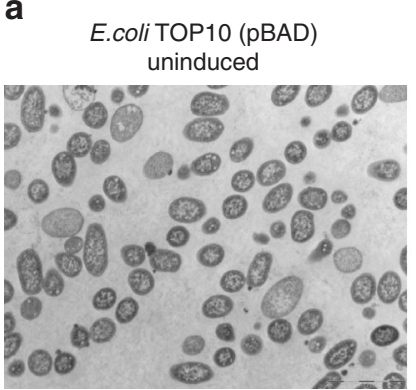

d

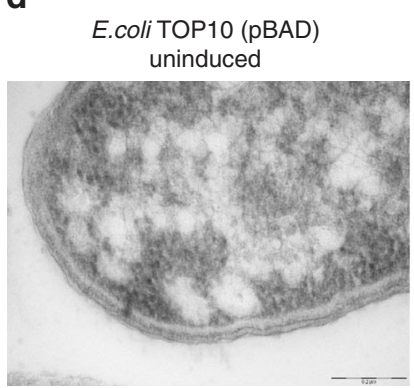

b

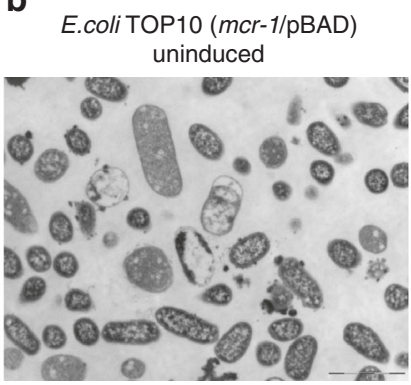

e

E.coli TOP10 (mcr-1/pBAD) uninduced

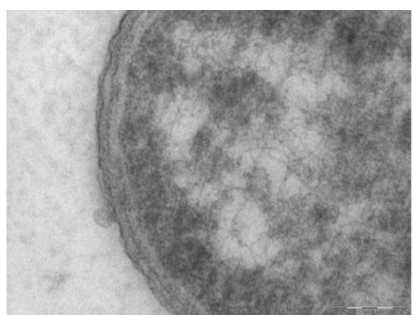

c

E.coli TOP10 (mcr-1/pBAD) induced $^{*}$

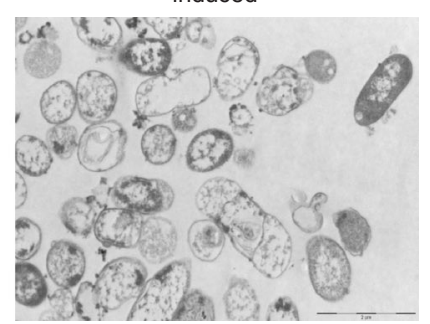

f

E.coli TOP10 (mcr-1/pBAD) induced* $^{*}$

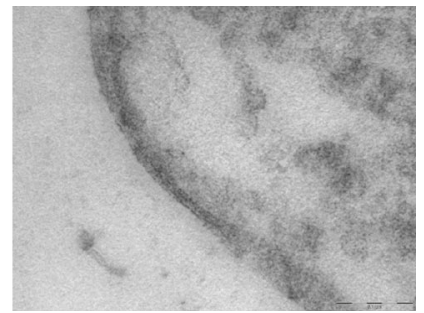

Fig. 3 TEM micrographs of untreated and treated E.coli. In $\mathbf{a}$ and $\mathbf{b}$, TEM micrographs of untreated control cells ( $E$. coli TOP10 with pBAD minus mcr-1, and $E$. coli TOP10 ( $m c r-1 / \mathrm{pBAD})$ without L-arabinose induction, respectively); both cells are intact with a well-defined inner and outer membrane, and showed a highly homogeneous electron density in cytoplasm region (d and $\mathbf{e}$ ). c TEM micrographs of mcr-1 overproducing cells; the damaging outer membrane and some completely lysed cells were observed (f)

a

E.coli TOP10 (mcr-1/pBAD)

uninduced

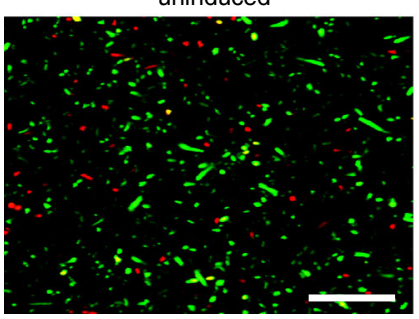

b

E.coli TOP10 (pBAD)

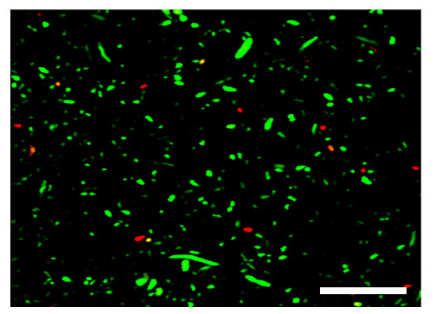

d

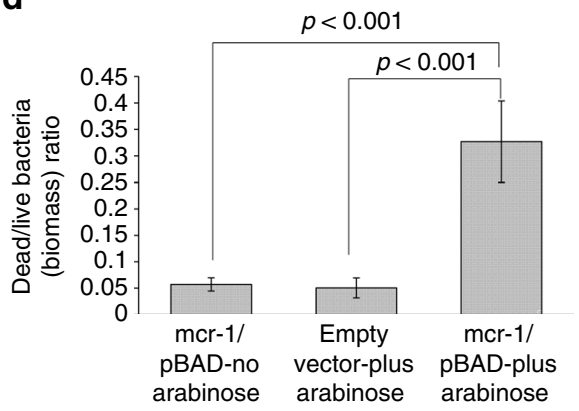

C E.coli TOP10 (mcr-1/pBAD)

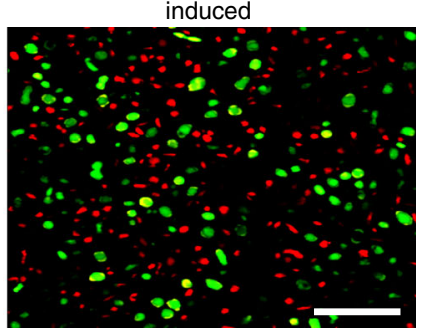

Fig. 4 The toxic effects of mcr-1 overexpression on cell viability. a-c Confocal laser scanning microscopy images of cells treated with/without L-arabinose and stained with LIVE/DEAD ${ }^{\circledR}(n=3)$. Live and dead cells presented green and red colour, respectively. Scale bar is $15 \mu \mathrm{m}$. $\mathbf{d}$ Ratio of dead to live bacteria (biomass) obtained from CLSM z-stack images through COMSTAT analysis of E. coli biofilms grown for $16 \mathrm{~h}$ in LB broth, followed by $\pm \mathrm{L}$-arabinose (0.2\% $\mathrm{W} / \mathrm{v} ; 8 \mathrm{~h})$ treatment, where the biofilms were stained with LIVE/DEAD ${ }^{\circledR}(n=4)$. The COMSTAT data was assessed using one-way analysis of variance (ANOVA) followed by Tukey-Kramer multiple comparisons post hoc test. Statistical significance was set at $p<0.05$

induction) (Fig. 4a, b). COMSTAT analysis of the CLSM biofilm images revealed that relative dead/live biomass ratio (Fig. $4 \mathrm{~d}$ ) of induced E. coli TOP10 ( $m c r-1 / \mathrm{pBAD})$ was at least six times higher than $E$. coli TOP10 with pBAD minus $m c r-1$, and E. coli TOP10 ( $m c r-1 / \mathrm{pBAD})$ minus arabinose ( 0.33 vs $0.06 ; p<0.001$, using one-way analysis of variance (ANOVA)). Morphological alterations were also apparent in CLSM imaging, where the live cells (green) in Fig. 3c appeared more spherical and 'bloated', in keeping with the changes observed in the TEM studies (Fig. 3c). Overexpression of $b l_{\mathrm{TEM}-1 \mathrm{~b}}$ showed no evidence of increased cell death compared to that of $E$. coli TOP10 with pBAD minus $m c r-$ 1, and E. coli TOP10 ( $m c r-1 / \mathrm{pBAD}$ minus L-arabinose induction 
a

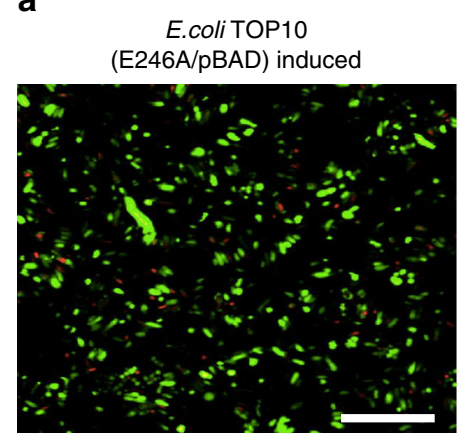

d

E.coli TOP10 (E246A/pBAD) uninduced

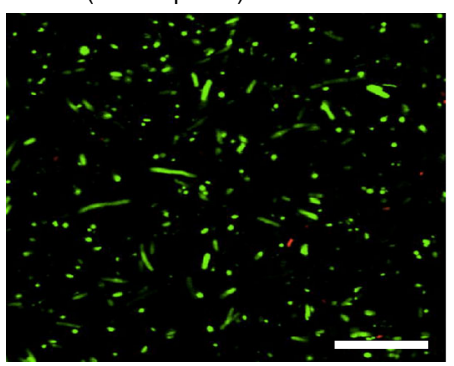

b

E.coli TOP10 (mcr-1 soluble/pBAD) induced

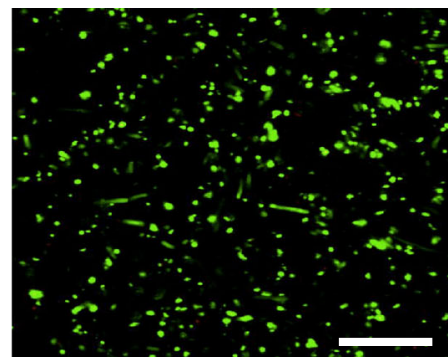

e E.coli TOP10
(mcr-1 soluble/pBAD) uninduced

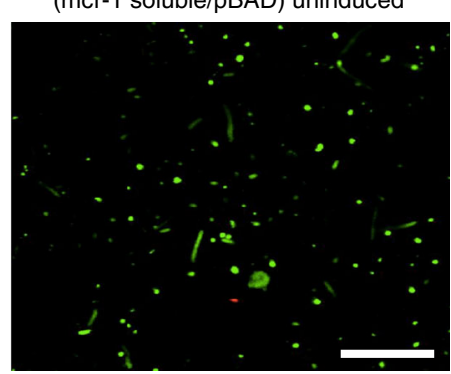

C

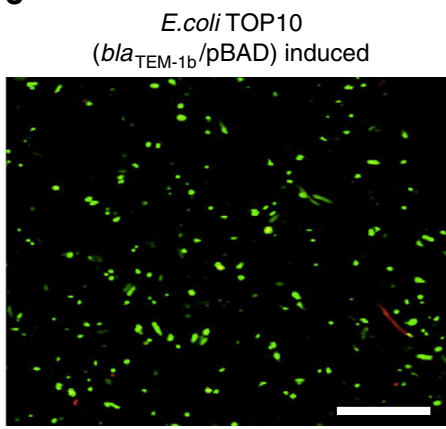

$\mathbf{f}$

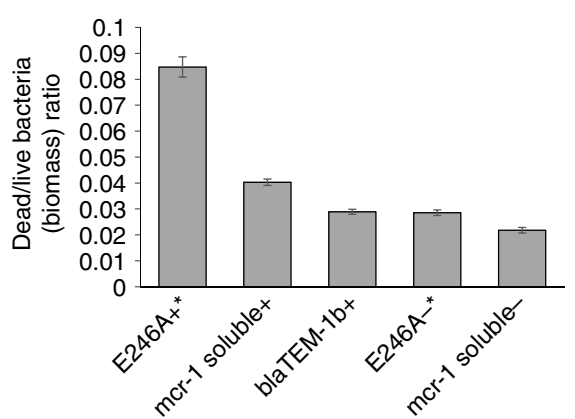

Fig. 5 The toxic effects of $m c r-1$ mutants on cell viability. a-c Confocal laser scanning microscopy images of cells treated with L-arabinose ( $0.2 \%$ w/v; $8 \mathrm{~h}$ ) and stained with LIVE/DEAD ${ }^{\circledR}(n=4)$. Live and dead cells presented in green and red colour, respectively. $\mathbf{d}$ and e Confocal laser scanning microscopy images of control cells treated without L-arabinose for $8 \mathrm{~h}$ and stained with LIVE/DEAD $(n=4)$. Live and dead cells presented in green and red colour, respectively. $\mathbf{f}$ Ratio of dead to live bacteria (biomass) obtained from CLSM $z$-stack images through COMSTAT analysis $(n=4)$. The COMSTAT data was assessed using one-way analysis of variance (ANOVA) followed by Tukey-Kramer multiple comparisons post hoc test, '+' and '-' indicate bacteria treated with or without L-arabinose, respectively

(Fig. 5c)) providing further evidence that the cellular effects seen with increased expression of MCR-1 are not due to the fitness burden of protein expression.

mcr-1-mediated membrane-bound resistance mechanism. Our previous study has shown that MCR-1 is a membrane-bound enzyme consisting of five hydrophobic transmembrane helixes and a soluble form located in the periplasmic domain ${ }^{9}$. To examine whether the transmembrane domain or MCR-1 catalytic domain (typified by aspartate at position 246) plays a role on cell integrity and bacterial fitness, MCR-1 (E246A), and a MCR-1 soluble domain (residues 219-541 and lacking the N-terminal membrane-bound region) mutant, were cloned into a pBAD-hisA plasmid. Both constructed plasmids were transferred into TOP10 cells and induced with $0.2 \%(\mathrm{w} / \mathrm{v})$ L-arabinose at exponential phase. Results show that increased expression of MCR-1 (E246A) produced a toxic effect on the $E$. coli cells as evidenced by decreased growth rate (Supplementary Fig. 4), significant membrane degradation (Supplementary Fig. 5) and moderate fitness loss (Fig. 6a). In contrast, the increased expression of the MCR-1 soluble domain (219-451) did not show any marked changes in growth rate, fitness loss or membrane architecture (Fig. 5b, f, Fig. 6a, and Supplementary Figs. 4 and 5). However, the toxic effects of the MCR-1 (E246A) mutant is moderate, compared to the marked effect of MCR-1 wild-type (Figs. 2-4). For example, increased expression of the full-length $m c r-1$ gene caused at least three times higher bacterial death than overproducing incomplete MCR-1 (E246A) (dead/live bacteria ratio, 0.33 vs 0.09) (Fig. 5a, f). Therefore, our data indicate that the fitness loss and the destruction of the membrane architecture is due to both the embedding of the protein in the E. coli outer membrane and the phosphoethanolamine modification of LPS. Phosphoethanolamine addition on lipid A in MCRPEC has been observed by ESIQTOF/MS (Supplementary Fig. 6), compared to that of nonMCRPEC.

mcr-1-mediated LPS modification reduced the stimulation of macrophage. $m c r-1$ encodes an enzyme that catalyses the transfer of phosphoethanolamine onto a phosphate of the $\mathrm{N}$-acetylglucosamine head group of lipid A, and its expression affects cell morphology (Fig. 3) and survival (Fig. 4). Therefore, to investigate whether the modified LPS of MCRPEC alters the activation of human macrophage THP-1, LPS-mediated human macrophage stimulation assays were undertaken. The macrophages were activated by serial concentrations (4.5, 0.45, 0.045 and $0.0045 \mathrm{ng}$ per $\mathrm{ml}$ ) of LPS extracted from $E$. coli TOP10 with pBAD minus $m c r-1$, and E. coli TOP10 ( $m c r-1 /$ pBAD plus $0.2 \%$ $\mathrm{L}$-arabinose). The production of interleukin (IL) -6 and tumour necrosis factor (TNF) were assayed by using DuoSet ${ }^{\circledR}$ ELISA kit ( $R \& D$ systems, UK). The concentrations of IL-6 produced by macrophages induced by unmodified LPS were consistently higher than IL-6 levels produced by MCR-1 modified LPS at 8 and $24 \mathrm{~h}$ (Fig. 7a, b). Additionally, TNF-alpha levels were also higher in macrophages stimulated by unmodified LPS than compared to modified LPS (Fig. 7c).

Acquisition and stability of high-level colistin resistance mutants. MCRPEC was first discovered on the premise that $E$. coli can rarely acquire colistin resistance by chromosomal mutations alone and that the levels mediated by $m c r-1$ are moderate compared with other mechanisms ${ }^{1,2,9}$. Data 
a

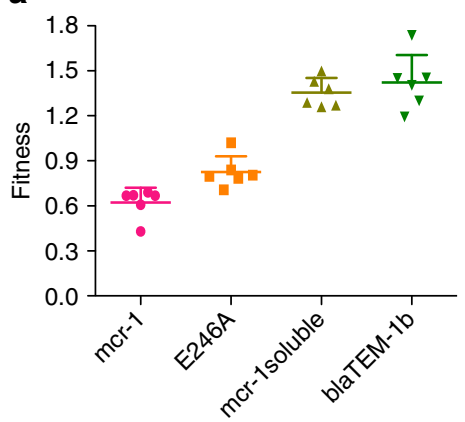

d

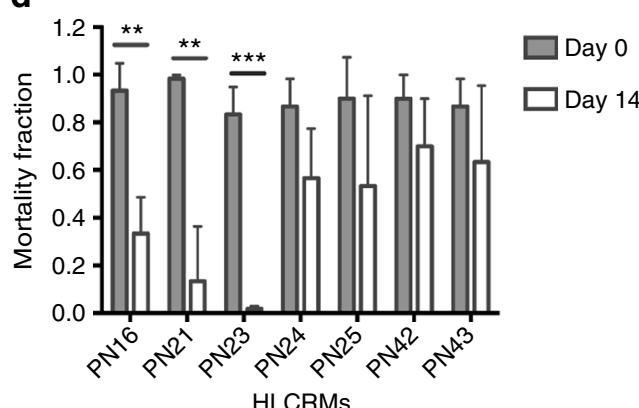

b

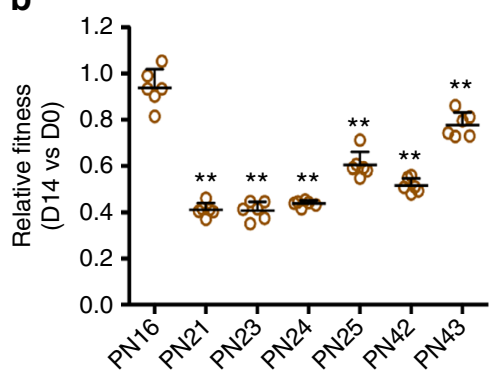

Day 14-HLCRMs c

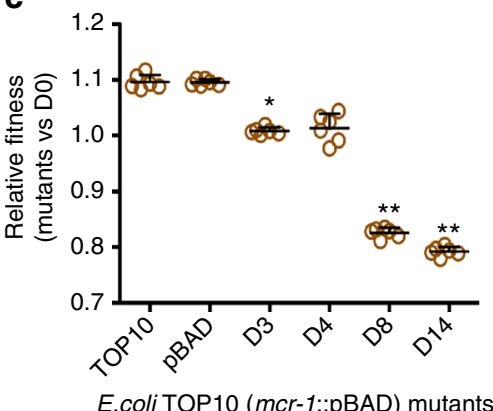

Fig. 6 Competition assays and G. mellonella killing models in HLCRMs. a Fitness of full-length mcr-1, calalytically inactivated mcr-1(E246A), and mcr-1 soluble domain. Fitness of blaTEM-1b as a negative control. $\mathbf{b}$ Relative fitness of wild-type mcr-1-positive strains and their derivatives. $\mathbf{c}$ Relative fitness of mcr-1/pBAD-positive E.coli TOP10 strains and their derivatives. In all fitness figures, error bars represent the SD $(n=6)$. The differences in fitness were tested using non-parametric Mann-Whitney test, ${ }^{*}$ indicates $0.01<p$ value $<0.05$ and ${ }^{* \star}$ indicates $p$ value $<0.01$. The average relative fitness and $p$ values are listed in Supplementary Table 6. d HLCRMs displays impaired virulence in the G. mellonella infection models. All results represent means of three independent experiments with 10 larvae per treatment. Mortality bar charts were plotted using the Kaplan-Meier method (GraphPad Software). Error bars represent the SD $(n=3)$ and $p$ value for strains PN16 $(p=0.0056, t=5.427, \mathrm{~d} . \mathrm{f}=4), \mathrm{PN} 21(p=0.0029, t=6.509, \mathrm{~d} . \mathrm{f}=4)$ and PN23 $(p=0.0003, t=$ $2.183, \mathrm{~d} . \mathrm{f}=4$ ) were calculated by student $t$ test. ${ }^{\star \star}$ indicates $0.001<p$ value $<0.01$, ${ }^{\star \star \star}$ indicates $p$ value $<0.001$

a

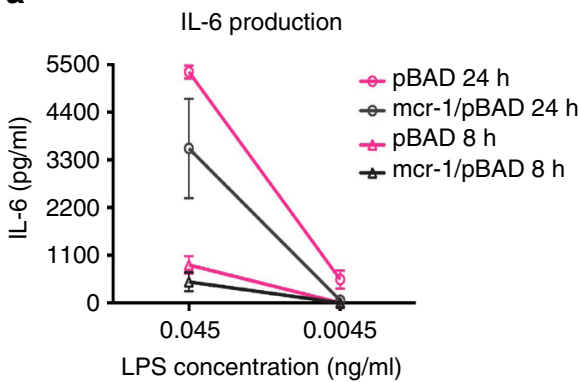

b

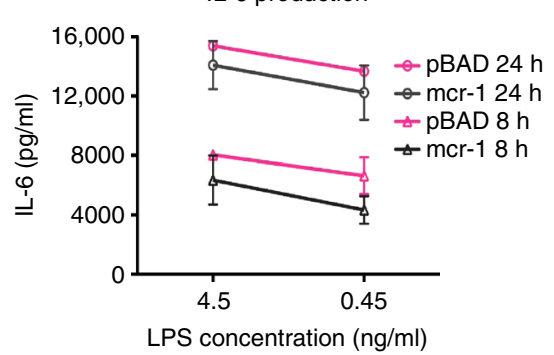

C

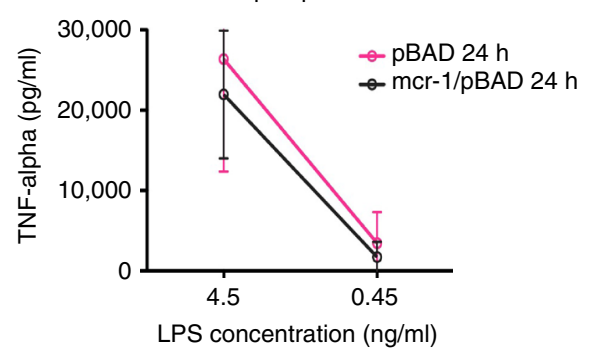

Fig. 7 Expression of cytokines in LPS-mediated THP-1 macrophage. $\mathbf{a}$, $\mathbf{b}$ and $\mathbf{c}$ indicated IL-6 $(n=2)$ and TNF-alpha $(n=3)$ productions in the modified LPS (grey) or normal LPS (red)-treated cell culture, respectively. Error bars represented SD

previously published by us would suggest that the level of in vivo colistin resistance mediated by MCRPEC can protect the cells compared to control strains ${ }^{9}$. However, the data from Figs. 2-4 would suggest that the expression of $m c r-1$ is also very tightly controlled because of the fitness cost of increased $\mathrm{mcr}-1$ expression. We explored whether MCRPEC could generate high-level colistin resistance mutants (HLCRMs) over nonMCRPECs and investigated how damaging these changes would be. HLCRMs were generated from the seven wild-type MCRPEC strains described in Supplementary Table 1. These isolates possessed low-level resistance to colistin (4-8 $\mathrm{mg} \mathrm{l}^{-1}$ ), and HLCRMs were generated through exposure to increasing concentrations of colistin. After a 14-day serial colistin challenge, all seven wild-type MCRPEC strains exhibited an increase in colistin resistance with a four to 64 -fold increase in colistin
MICs (Supplementary Fig. 7A and Supplementary Table 4). The rate of increase and final level of resistance varied considerably, with PN16 and PN23 reaching $256 \mathrm{mgl}^{-1}$; PN21, PN25 and PN43 reaching $128 \mathrm{mg} \mathrm{l}^{-1}$; PN42 reaching $32 \mathrm{mgl}^{-1}$, yet PN24 could be increased to $16 \mathrm{mg} \mathrm{l}^{-1}$.

We also exposed E. coli TOP10 ( $m c r-1 / \mathrm{pBAD})$, E. coli TOP10 (pBAD) and E. coli TOP10 to the same level of passaging (Supplementary Fig. 7B and Supplementary Table 5). E. coli TOP10 ( $m c r-1 / \mathrm{pBAD}$ ) (in the absence of arabinose) displayed a 64-fold (from 0.5 to $32 \mathrm{mg} \mathrm{l}^{-1}$ ) increase in colistin MICs. However, both E. coli TOP10 (pBAD) and E. coli TOP10 did not show any elevation in colistin MICs remaining at $0.125 \mathrm{mg} \mathrm{l}^{-1} \pm 1$ dilution over the 14-day challenge. These data indicate that the increase in colistin resistance shown by $E$. coli TOP10 ( $m c r-1 / \mathrm{pBAD})$ is singularly due to the presence of $m c r-1$. Both the expression level and copy number 
a

G. mellonella killing model

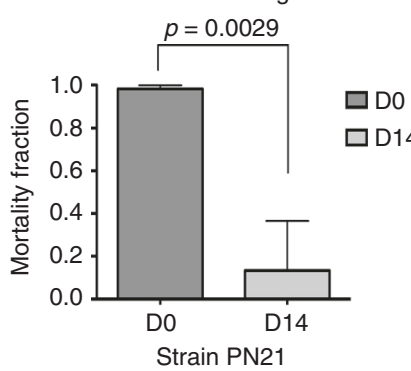

b

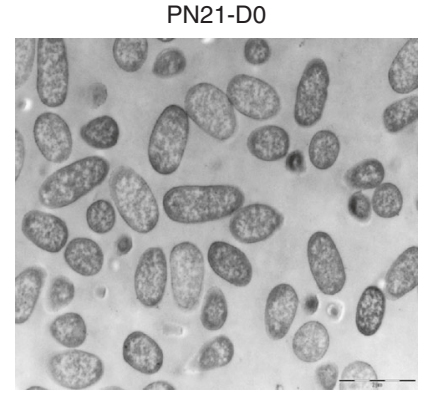

C

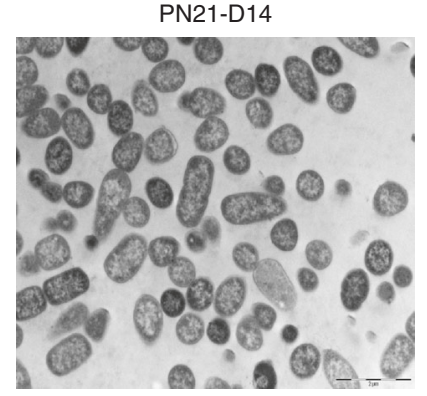

Fig. 8 G. mellonella killing data and TEM micrographs of strain PN21. a G.mellonella mortality rate in PN21 parental strain (D0) and its mutant (D14). Error bars represent the SD $(n=3)$ and $p$ value was calculated by Student's $t$ test $(t=6.509, d . f=4)$. $\mathbf{b}$ and $\mathbf{c}$ indicated TEM micrographs of parental strain PN21 (D0) and PN21(D14) mutant, respectively. Membranes for both strains are intact with a highly homogeneous electron density in cytoplasm region

of $m c r-1$ gene were determined by qRT-PCR for all MCRPEC isolates (Supplementary Fig. 8 and Supplementary Table 1). Compared with the wild-type strains, no significant difference in plasmid copy number and $m c r-1$ expression could be observed in the corresponding HLCRMs except for MRCPECs isolates PN21 and PN25, where the expression of $m c r-1$ has increased by $\sim 11$-fold and threefold, respectively (Supplementary Fig. 8).

Given the fitness cost of $m c r-1$ to $E$. coli and the fact that the HLCRMs are likely to mediate higher resistance in vivo and be more problematic to treat, the stability of these HLCRMs needed to be assessed for their high-level colistin resistance stability. Accordingly, the 14-day challenged HLCRMs were subsequently serially passaged in colistin-free medium to determine if the highlevel colistin-resistance phenotype could be reversed. Three MCRPECs, PN24, PN25 and PN42, showed the same level of colistin resistance as their respective HLCRMs (day-14 mutants), after 14-day passages in lysogeny broth (LB) broth without colistin indicating that the high-level of colistin resistance was very stable (Supplementary Fig. 7C). However, MCRPECs PN16, PN21, PN23 and PN43 showed a marked decreased in colistin resistance during passaging in antibiotic-free medium with HLCRM PN43 being the most unstable (highest loss of colistin resistant population) followed by HLCRM derived from isolates PN23, PN16 and PN21 (Supplementary Fig. 7C).

In vitro relative fitness of HLCRMs. Our data examining the physiological burden of $m c r-1$ would suggest that the HLCRMs might even be defective in fitness and pathogenicity. Therefore, to compare the growth rates of the wild-type MCRPEC isolates with their respective HLCRMs, we measured growth over a serial time course of HLCRMs strains that showed different resistance levels of resistance to colistin at day $0,3,7,11$ and 14 . Most of the HLCRMs grew up to threefold slower than their wild-type parent isolates and, in particular, those mutants recovered at day 11 and day 14 (Supplementary Fig. 9A-G). The exception being PN16 where there was no significant difference in growth rate between the parent and its corresponding HLCRM (even day-14 mutant) (Supplementary Fig. 9A). Additionally, we also examined the growth curves of HLCRMs generated from E. coli TOP10 ( $\mathrm{mcr}-1 /$ pBAD). Similarly, day- 8 and day-14 mutants (both MICs of 16 and $32 \mathrm{mgl}^{-1}$ for colistin, respectively) showed a decreased growth compared to that of their parental strains (Supplementary Fig. 9H).

Based on our data from bacterial growth curves, we propose there is substantial fitness disadvantage to the HLCRMs when compared to their MCRPEC parent strains. To test this hypothesis, in vitro competition assays were performed and HLCRMs were directly competed against their MCRPEC parental strains. The fitness of the MRCPEC parent strains and HLCRMs were relative to the growth of the control strain that was fixed at
1.0 (Methods) (Fig. 6b). Most (6/7) of the day-14 HLCRMs had lower competitive fitness than that of their corresponding parent strains (Fig. 6b) with HLCRMs derived from PN21, PN23, PN24, PN25 and PN42 possessing a relative fitness of $0.4-0.6$ and HLCRM PN43 and relative fitness rate of 0.78 (Mann-Whitney test-corrected $p=0.0022$ ) (Fig. 8a and Supplementary Table 6). The PN21 and PN23 mutants, the former has 11-fold increased mcr-1 gene expression (Supplementary Fig. 8), were associated with the highest fitness burden $(\sim 0.41, p=0.0022)$ (Fig. $6 \mathrm{~b}$ and Supplementary Table 6). The exception was HLCRM derived from PN16 that had a fitness rate similar to its parent strain $(\sim 0.94, p=0.1320)$ (Fig. 6b and Supplementary Table 6). We also examined the fitness cost for E. coli TOP10 ( $m c r-1 / \mathrm{pBAD})$, E. coli TOP10 (pBAD) and E. coli TOP10 (Fig. 6c). Day 3 and 4 HLCRMs of E. coli TOP10 ( $m c r-1 / \mathrm{pBAD}$ ) had slightly less fitness (1.0 vs 1.1 of E. coli TOP10 (pBAD) and E. coli TOP10) and day 8 and 14 were less fit ( 0.83 vs 1.1 , and 0.79 vs 1.1 , respectively) (Fig. 6c). Generally, our data show a significant fitness burden with HLCRMs compared to their MCRPEC parent strains.

Virulence reduction of MCRPEC and HLCRMs. To determine whether the level of virulence of HLCRMs have been reduced compared to the MCRPEC parental strains, we used Galleria mellonella as a model of infection ${ }^{24,25}$. Larvae were infected with each of the MCRPEC parental strains and their respective HLCRMs, with differing bacterium inoculums in order to illicit appropriate live and dead larvae populations. The mortality of larvae was dependent on the number of bacteria injected (data not shown) and this was varied to show the greatest effect with each parent/mutant pair. As shown in Fig. 6d and Supplementary Fig. 10, a significant decrease in G. mellonella killing was observed in all HLCRMs, compared to their respective wild-type parent strains. In particular, HLCRMs derived from MCRPECs PN16, PN21 and PN23 gave mortality fractions of $0.33(p=0.0056$, Student's $t$ test), $0.13(p=0.0029)$ and $0.02(p=0.0003)$, respectively, compared to their respective parent strains that were between 0.8 and 1.0. Because the MCRPEC parental strains are highly heterogeneous with respect to their genomic background, the infection of $G$. mellonella resulted in different larvae survival rates (Supplementary Fig. 10). However, when we compared the morphological changes using TEM analysis, there was no significant change between mutants and their corresponding MRCPEC parents (Fig. 8b, c), even there was a decrease in $G$. mellonella killing $(p=0.0029)$ exhibited in PN21 (Fig. 8a).

In wild-type strains, the level of colistin resistance mediated by $m c r-1$ gene is often moderate $\left(2-8 \mathrm{mg} \mathrm{l}^{-1}\right)$. The in vitro generated HLCRMs possessed reduced fitness and virulence. We also determined that even the low level of colistin resistance mediated by $m c r-1$ gene in clinical strains attenuates bacterial virulence in 
a

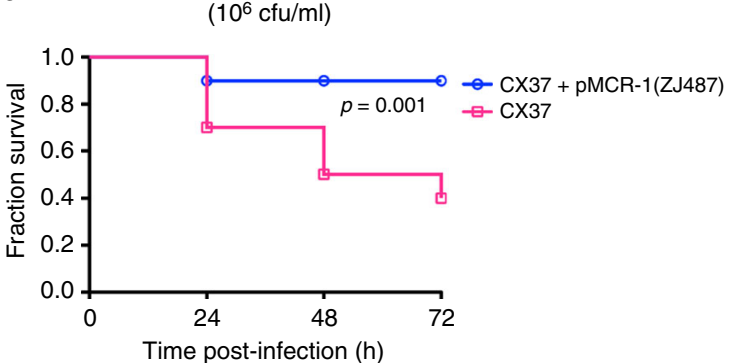

C

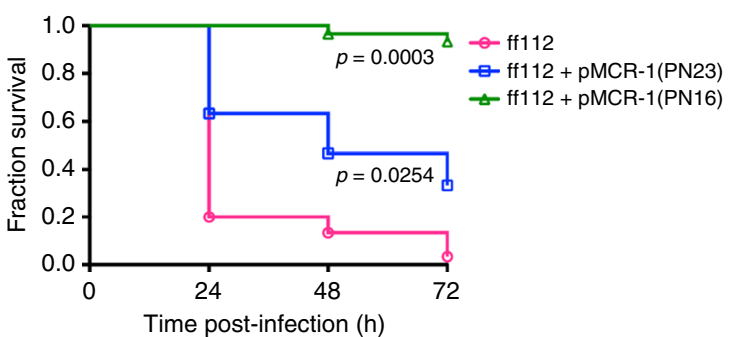

b

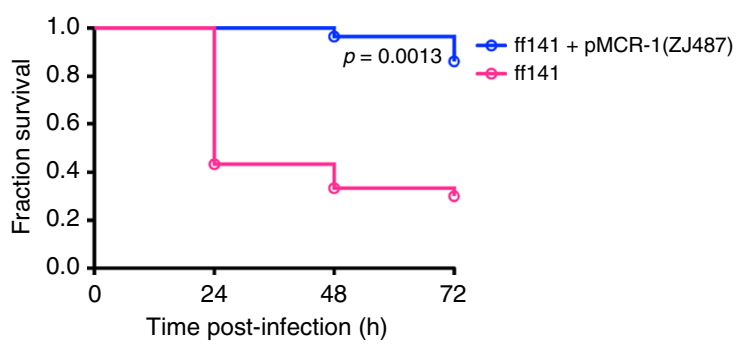

d

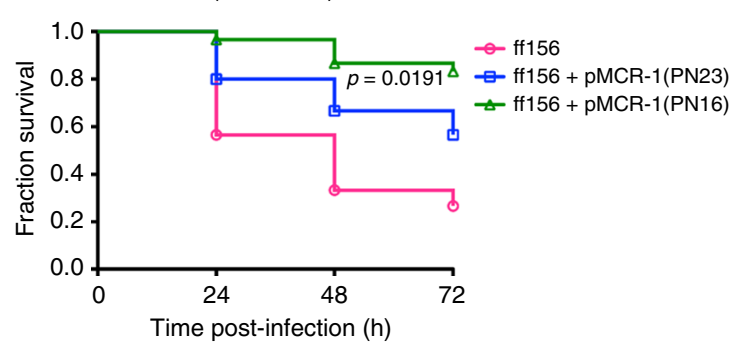

Fig. 9 Kaplan-Meier plots showing the percent survival of $G$. mellonella over $72 \mathrm{~h}$ post infection with MCRPEC and non-MCRPEC human clinical strains. Survival curves were plotted using the Kaplan-Meier method (GraphPad Software). Error bars represent the SD $(n=3)$ and $p$ value for a $(t=8.654, \mathrm{~d} . \mathrm{f}=$ $4)$, b $(t=8.050$, d.f $=4)$, $\mathbf{c}(t=12.07, \mathrm{~d} . \mathrm{f}=4$ for strain ff112 with pMCR-1(PN16) and $t=3.479, \mathrm{~d} . \mathrm{f}=4$ for strain ff112 with pMCR-1(PN23)) and $\mathbf{d}(t=$ 3.801, d.f $=4$ ) were calculated by Student's $t$ test

the G. mellonella infection model. We chose four non-MCRPEC clinical strains, belonging to different ST types: ST638 (ff112), ST589 (ff141), ST127 (ff156) and ST648 (CX37), which is one of diverse ST groups associated with $m c r-1$ gene in China ${ }^{10}$. Three different $m c r-1$ plasmids isolated from MCRPEC strains (PN16, PN23 and ZJ487) were transferred to these non-MCRPEC strains by electroporation. We have shown that the acquisition of $\mathrm{mcr}-1$ naturally occurring plasmids do not affect bacterial growth of the host (wild-type clinical strains) strain and so their fitness cost is modest (Supplementary Fig. 11). However, their virulence is markedly depleted (Fig. 9) supporting our hypothesis that the loss of virulence shown by MCRPEC is not due to growth rate but by the activity of $m c r-1$ on the E. coli outer membrane (Fig. 3).

Sequencing analysis of $\boldsymbol{m} \boldsymbol{c r}-\mathbf{1}$ genetic contexts. To elucidate genetic changes during the evolution of high levels of colistin resistance, whole-genomic sequencing was performed on all seven wild-type parental strains (day 0) and their isogenic HLCRMs (day 14). For each strain, a read coverage of at least 80 -fold was generated and trimmed using Trim Galore and the genomes were de novo-assembled into contigs using SPAdes (3.9.0) with predefined kmers set (Methods). When comparing genomes with their parental strains, the seven HLCRMs showed no amino acid mutations in $\mathrm{mcr}$-1-carring contigs including their promoter and the immediate surrounding genetic contexts. In addition, they all possess $m c r$-1-carrying plasmids, exception of strain PN43, which $m c r-1$ gene located in chromosome (Supplementary Table 1 and Supplementary Fig. 5). As shown in Supplementary Fig. 1A, there are three representative genetic contexts of $m c r-1$-carrying contigs were identified in seven MCRPEC. Firstly, the $m c r-1$ identified on chromosome in PN43 isolate was flanked directly by ISapl1, the genetic context of which is identical to other chromosomal $\mathrm{mcr}-1$ gene (accession number: KY421935, LT594504 and ENQ00000000), except that a flanking hypothetical open-reading frame is truncated by IS1294. Of note, in two IncI2 plasmids from PN16 and PN21 isolates, the genetic context of $m c r-1$ are identical to pHNSHP45 (accession number: KP347127) harbouring
IncI2 scaffold and type VI secretion system components (Supplementary Fig. 1B). The rest of four $m c r-1$ positive IncX4 plasmids from PN23, PN24, PN25 and PN42 isolates exhibit remarkable similarity to the backbone of pMCR-NJ-IncX4 (accession number: KX447768) (Supplementary Fig. 1C).

\section{Discussion}

Acquiring antibiotic resistance by mutation or horizontal gene transfer tends to be associated with a fitness $\operatorname{cost}^{26-28}$. This cost plays a key in limiting the spread and maintenance of resistance in pathogen populations by generating selection against resistant strains under conditions where antibiotic doses are low, for example, during transmission between hosts ${ }^{28,29}$. However, recent studies have challenged this paradigm and provide evidence supporting the notion that, at least in some systems, the evolution of resistance can be associated with fitness advantages including the enhanced ability to cause disease $e^{30,31,32}$. We have shown that the case for $m c r-1$ imposing a fitness cost on its $E$. coli host is substantial and increased expression of $m c r-1$ not only impairs cell growth (Fig. 2a) and diminishes bacterial fitness (Fig. 2c), but also alters the cell's architecture (Fig. 3c) and kills the bacteria (Fig. 4c). Evolving colistin resistance by acquiring $m c r-1$ therefore challenges bacterial populations with an evolutionary trade-off: high-level expression of $m c r-1$ provide protection against the antibiotic, but this increase in resistance compromises growth rate, fitness, membrane structural integrity and increases cellular death. This trade-off may explain several important phenomena. First, $m c r-1$-mediated colistin MICs are at best moderate (usually 2-8 $\mathrm{mg} \mathrm{l}^{-1}$ ) when compared to colistin resistance $\left(8-256 \mathrm{mg} \mathrm{l}^{-1}\right)$ mediated by $p m r A / p m r B^{3,9,10,16}$. Secondly, we show that $m c r-1$ plasmid copy number is very low when compared to other plasmid-mediated mechanisms, eg, IncA/C plasmids carrying $b l a_{\mathrm{NDM}-1}$ and this appears to protect the cell from too many copies of $m c r-1^{21,33}$.

As a negative control, we show that the high expression of $b l a_{\mathrm{TEM}-1}$ has little, if any, effect on $E$. coli fitness and survival 
(Fig. 5c, Supplementary Figs. 3C and 4C), and therefore the effects witnessed with MCR-1 are due to its incorporation into the E. coli membrane or by its catalytic action-the phosphoethanolamine modification of LPS. It is interesting to note that only a few mobile membrane-associated antibiotic resistance mechanisms have been characterised. One of them is the tetracycline efflux system, eg, tet $A(B)$, which is tightly regulated by its neighbouring gene, tet $R$. It has been shown that overproduction of tetA is lethal, which is probably due to its effect on the membrane topology $y^{34-36}$. Unlike the tet $A$-like efflux pump system, $m c r-1$ does not possess an adjacent repressor to control its expression and its expression is controlled by other regulatory systems such as low plasmid copy number. Compared with the considerable burden of high expression of MCR-1 (Figs. 2-4), the toxic effects of its two derivatives, MCR-1 E246A and MCR-1 soluble region, are moderate, which can be observed by some restoration of its outer membrane integrity and a reduction in death rate (Fig. 5, Supplementary Figs. 3 and 4). However, as the toxic effects are more evident in MCR-1 E246A than the MCR-1 soluble region, MCR-1's cellular burden is due to both the embedding of the protein in the E. coli outer membrane and MCR-1's phosphoethanolamine modification of LPS.

China has now withdrawn colistin as an animal growth promoter and is now being deployed in human medicine ${ }^{4,37}$. However, the notion that MCRPEC will drastically diminish on withdrawal of colistin is parsimonious and naive. Our data previously, and the stability data on HLCRMs herein, suggests that colistin resistance in MCRPEC and HLCRMs can be stable ${ }^{9}$. Additionally, our data on E. coli TOP10 (mcr-1/pBAD) shows that HLCRMs can be generated from a lab-strain, but only after acquisition of $m c r$-1suggesting the increase in colistin resistance is not dependent on the strain background that is further supported by the fact that all our selected wild-type MRCPECs readily generated HLCRMs. Finally, it is possible that colistin-resistant strains will evolve compensatory adaptations that allow for $\mathrm{mcr}-1$ to be expressed at high levels at a low fitness cost. Compensatory adaptation is routinely detected in vitro ${ }^{28}$, and there is some evidence that compensatory adaptation maintains otherwise costly resistance mutations in vivo ${ }^{38}$.

Due to its central clinical role in causing Gram-negative bacterial sepsis, lipid A as an immune modulator has been thoroughly scrutinised ${ }^{39,40}$. However, despite the fact that the mechanisms of 4-amino-4-deoxy-L-arabinose and phosphoethanolamine LPS modification are well known, there have been very few systematic studies examining their impact on lipid A as an immune stimulant. Studies on Salmonella have examined the addition of phosphoethanolamine and found no significant change in virulence ${ }^{41}$. These data are supported by a recent study in Haemophilus ducreyi that used knockout deletions of phosphoethanolamine modifying genes ( $p t A, p t d A$ and $p t d B)$ and concluded that the triple mutant was a virulent as the parent ${ }^{42}$. A recent study by John et al. ${ }^{43}$ examined the phosphoethanolamine and its effect in Neisseria meningitidis by comparing invasive and carrier isolates and found more phosphoethanolamine and sialic acid substitutions from invasive strains suggesting that phosphoethanolamine-lipid A modification enhances virulence. This finding also has been identified in non-mor-1 mediated colistin resistance in K. pneumoniae, where lipid A remodelling mediated by the $\operatorname{mgr} B$ mutation, resulted in increased colistin resistance enhancing the virulence of $K$. pneumoniae by decreasing the affinity of colistin and attenuating host defence response ${ }^{44}$. These data are further supported by O'Brien and colleagues examining Campylobacter jejuni phosphoethanolamine modified lipid A, showed increased recognition of a human Toll-like receptor and increased commensal colonisation in mice ${ }^{45}$. However, Zughaier et al. ${ }^{46}$ examined $N$. gonorrhoeae phosphoethanolamine modified lipid A and showed that it reduced autophagy in human macrophages and useful mechanism to evade the host immune system. Thus far, there are no similar studies on $E$. coli and this is the first examining the effect of $m c r-1$ on $E$. coli fitness and virulence. The data presented in this study indicate that E. coli (in this case, E. coli TOP10 ( $m c r-1 /$ pBAD)) is forced to finely tune the expression of $m c r-1$ or else the overexpression becomes toxic resulting in profound changes in the architecture of the outer membrane (Fig. 3c), causing leakage of cellular cytoplasm (Fig. 3c) and death (Fig. 4c). Interestingly, in a HLCRM (PN21) where that was at least 11-fold expression on mcr-1 (Supplementary Fig. 8), we observed no cellular deterioration (Fig. 9b, c), suggesting that PN21 may have a different genetic makeup and is able to produce compensatory mutations ${ }^{47}$. When we have examined the immediate $m c r-1$ genetic context comparing HLCRMs and their respective wild-type parents, no changes in the promoter regions in any of our seven isogenic pairs could be identified (Supplementary Fig. 1). When compared to their respective wild-type parental strains, most of HLCRMs, except strains PN25 and PN42, showed no amino acid mutations in PmrE, PmrAB, PmrC, PhoPQ, MgrB, ArnB/D, CptA, EptB, IpxM, MicA and ArcAB, which are known to be associated with colistin resistance ${ }^{3,48}$. However, in strains PN25 and PN42, amino acid mutations have been identified in PmrA $(\mathrm{R} 81 \mathrm{~S})^{48}$ and $\mathrm{PmrB}(\mathrm{V} 161 \mathrm{M})^{49}$, respectively, which are responsible for reducing susceptibility of colistin. Another finding is that HLCRMs altered the susceptibility to other antimicrobials (Supplementary Table 7). HLCRMs have obtained higher MICs of colistin (16-256 $\mathrm{mg}^{-1}$ ) by multiple passages in the increasing concentrations of colistin. Conversely, these mutants are more susceptible to other antibiotics (Supplementary Table 7), for instance, expect strain PN24, the other six HLCMRs strains caused one to 16-fold MIC reduction of chloramphenicol, compared with their respective parental strains. The MICs of tigecycline, regarded as one of the last resorts to treat infections caused by multidrug resistance bacteria, have one to 32 -fold reduced in all HLCRMs. This is likely due to $m c r-1$-mediated membrane permeability in these strains.

As $m c r-1$ continues to spread globally, and the clinical impact is assessed, it will be interesting to examine how virulent MCRPEC compare with non-MCRPEC. In this study, mor-1 positive plasmids were transferred to non-MCRPEC clinical strains (ST638 (ff112), ST589 (ff141), ST127 (ff156) and ST648 (CX37)) by electroporation. We have shown that the growth rate of all $m c r$-1-positive transformants appears to be lower than that of their non-MCR parents, but the difference is not statistically significant (Supplementary Fig. 11). Compared to non-MCRPEC strains from phylogenetic group D (considered as second-most virulent ExPEC group), the mortality of G. mellonella showed a marked reduction after acquisition of a $m c r-1$-positive plasmid (Fig. 8). To conclude, it would appear the acquisition of $m c r-1$ by E. coli is a 'poisoned chalice'-on the one hand $m c r-1$ is required to provide protection in a colistin-rich environment, yet acquisition compromises the bacterium's normal physiology; furthermore, overexpression results in acute 'toxicity'.

\section{Methods}

Bacterial strains and growth conditions. Seven E. coli isolates carrying the $\mathrm{mcr}-1$ gene (MCRPEC) were isolated and characterised from Thailand and used as parental strains (Supplementary Table 1). Bacteria were grown in LB broth or chromogenic agar (Liofilchem, Roseto, Italy), supplemented with appropriate antibiotics at $37^{\circ} \mathrm{C}$. E. coli TOP10 ( $\left.\mathrm{mcr}-1 / \mathrm{pBAD}\right)$ was constructed by cloning the $m c r-1$ gene in-frame into low-copy number pBAD-hisA expression vector (ThermoFisher, UK) such that the expression of $m c r-1$ is controlled by the pBAD promoter of the $\operatorname{araBAD}$ (arabinaose) operon that can be regulated with L-arabinose induction ${ }^{50}$. Four non-MCRPEC clinical strains belonging to E. coli group D, 
were chosen as recipients for transformation by electroporation. All strains and plasmids used in this study are listed in Supplementary Table 2.

Plasmid constructs. An MCR-1 non-active enzyme was created by a single substitution (E246A). Its encoding gene was excised from $m c r-1$-pUC19 (E246A) ${ }^{19}$ and sub-cloned into plasmid pBAD-HisA to generate E246A/pBAD. To test the effect of the MCR-1 soluble domain on bacterial growth, the gene encoding the MCR1 soluble domain (lacking the five predicted transmembrane helices; codons 219-541) was cloned into pBAD-HisA vector with forward and reverse primers ( $m c r-1 \mathrm{~F}$ soluble and $m c r-1 \mathrm{R}$ soluble, Supplementary Table 3$)$. As a negative control, a B-lactamase gene, $b l a_{\text {TEM-1b }}$, a 861 bp fragment were generated by PCR with forward and reverse primers (Supplementary Table 3) and then inserted into pBAD-HisA vector using the restriction sites EcoRI and KpnI. All resultant plasmids were transformed into E. coli TOP10 cell (Invitrogen, UK), purified and its integrity confirmed by PCR, double restriction digestion and DNA sequencing.

Electroporation. Overnight cultures were diluted (1:50) in $5 \mathrm{ml}$ fresh $\mathrm{LB}$ broth and incubated at $37^{\circ} \mathrm{C}\left(220\right.$ r.p.m.) until the cultures reached an $\mathrm{OD}_{600}$ value of $0.5-0.7$. Bacterial cell cultures were kept on ice for $20 \mathrm{~min}$, followed by centrifugation at 5000 r.p.m. $(14,000 \times g), 4^{\circ} \mathrm{C}$ for $15 \mathrm{~min}$. The pellets were then resuspended in $1 \mathrm{ml}$ chilled $10 \%$ glycerol and re-centrifuged for $15 \mathrm{~min}$ at $4{ }^{\circ} \mathrm{C}$. After another two washes in chilled $10 \%$ glycerol, supernatants were discarded and the pellets were suspended in the residual glycerol. Subsequently, 50-100 ng of plasmid DNA were added into $50 \mu \mathrm{l}$ of electrocompetent cells and then the DNA cell mixture was transferred into a chilled cuvette. EC3 $(3.0 \mathrm{kv}, 5.5 \mathrm{~ms})$ programme was used for electroporation (Bio-Rad MicroPulser, France). Immediately, $950 \mu \mathrm{l}$ of warm LB broth was added to the cuvette and thoroughly mixed, followed by incubation at 37 ${ }^{\circ} \mathrm{C}$ for $1 \mathrm{~h}$, shaking vigorously at 220 r.p.m. A total of $100 \mu \mathrm{l}$ aliquot were plated onto a pre-warmed selective plate and incubated overnight at $37^{\circ} \mathrm{C}$.

Quantitative real-time PCR. Expression of $m c r-1$ was assayed by a two-step qRTPCR using primers $m c r-1-\mathrm{qF}, m c r-1-\mathrm{qR}$ and $m c r-1$ probe, with Precision $2 \times \mathrm{qPCR}$ Mastermix (PrimerDesign, UK) following manufacturers' protocol. Total RNA was extracted from bacteria using the RNeasy Plus kit with on column DNase digestion (Qiagen, Germany), followed by complementary DNA synthesis with DNAintegrated genomic DNA (gDNA) removal using QuantiTect Reverse Transcription kit (Qiagen, Germany), according to manufacturers' protocol. The absence of carry-over gDNA was verified for every experiment by comparative qPCR (or standard PCR) in the absence of reverse transcriptase. $r p o B$ expression level was used as internal control using primers $r p o B-\mathrm{qF}, r p o B-\mathrm{qR}$ and $r p o B$ probe. Relative expression results were obtained by the $\Delta \Delta C T$ analysis method using mean $C T$ value. For details of used primers and probes, see Supplementary Table 3.

For qPCR determination of $m c r-1$ copy numbers per cell, 0.1 ng of total gDNA was used as template with primers $m c r-1 \mathrm{qF}, m c r-1 \mathrm{qR}$ and $m c r-1$ probe and $16 \mathrm{~S}$ primers and probe (Supplementary Table 3). In parallel standard curves for $m c r-1$, $16 \mathrm{~S}$ were obtained using as template serial dilutions of $m c r-1$-carring plasmid DNA extracted from pSU18- $m c r-1$ strain ${ }^{19}$ (4.3 pg of DNA corresponding to $10^{6}$ copies, calculated through the website: http://cels.uri.edu/gsc/cndna.html) and E. coli TOP10 (Invitrogen) total gDNA (5 ng corresponding to $10^{6}$ cells) ${ }^{21}$, respectively.

\section{Selection of induced high-level colistin-resistant mutants. HLCRMs were} generated from seven wild-type $m c r-1$ positive strains through 14-day serial passaging with increasing concentrations of colistin (Alfa Aesar, US). Overnight cultures of parent strains were diluted to $10^{5}$ c.f.u. per $\mathrm{ml}$ and challenged with different concentrations of colistin (from 0.125 to $256 \mathrm{mg} \mathrm{l}^{-1}$ ) for $18-20 \mathrm{~h}$ at $37^{\circ} \mathrm{C}$. The next day, cultures in the last well that yielded visible bacterial growth were mixed with the first clear well (normally registered as the MIC) and challenged with colistin as described above. Cultures at 3, 7, 11 and 14 days were retained and stored at $-80^{\circ} \mathrm{C}$ for further analysis.

Stability of high-level colistin resistance in HLCRMs. To detect whether the colistin resistance in HLCRMs is stable, ie, reversible or not, serial passages of HLCRMs were performed in colistin-free medium. Overnight cultures of HLCRMs were diluted (1:500) into fresh LB broth without colistin and incubated with vigorous shaking ( 220 r.p.m.) for 18 h. To measure the proportion of colistin resistance bacterial population during reversion, overnight cultures were serial diluted, then inoculated on two types of chromogenic agar: one type is free of antibiotics, the other is containing various concentrations $\left(8,16,32,64,128,256 \mathrm{mg} \mathrm{l}^{-1}\right)$ of colistin depending on the level of colistin resistance mediated by the HLCRM. The c.f.u. per $\mathrm{ml}$ of colistin resistance cells were counted after $18-22 \mathrm{~h}$ incubation at $37^{\circ} \mathrm{C}$.

\section{Confocal laser scanning microscopy imaging using LIVE/DEAD staining. $E$.} coli TOP10 ( $m c r-1 / \mathrm{pBAD})$, E. coli TOP10 (pBAD only) and E. coli TOP10 $(n=4)$ were grown overnight in LB broth supplemented with $100 \mathrm{mg} \mathrm{l}^{-1}$ ampicillin (Fisher Chemical, UK) at $37^{\circ} \mathrm{C}$ (120 r.p.m.). Overnight cultures were standardised to $\mathrm{OD}_{600} 0.05$ and inoculated $(1: 10 ; \mathrm{v} / \mathrm{v})$ into 96 -well glass-bottomed plates (Whatman $\left.{ }^{\circledR}, \mathrm{UK}\right)$ in LB broth for $16 \mathrm{~h}\left(37^{\circ} \mathrm{C} ; 30\right.$ r.p.m.). The supernatant was gently removed and the biofilms were further incubated in fresh LB broth $\pm \mathrm{L}$ arabinose $(0.2 \%, \mathrm{w} / \mathrm{v})$ for $8 \mathrm{~h}$. The supernatant was removed and the biofilms stained with $6 \%$ LIVE/DEAD ${ }^{\circledast}$ (v/v; BacLight ${ }^{\text {TM }}$ Bacterial Viability Kit, Invitrogen) in phosphate buffered saline (PBS) prior to CLSM imaging (Leica TCS SP5) with ax63 lens. The CLSM $z$-stack images were analysed using COMSTAT image analysis software for quantification of biofilm biomass ${ }^{51}$. The COMSTAT data was assessed using one-way ANOVA followed by Tukey-Kramer Multiple comparisons post hoc test. Statistical significance was set at $p<0.05$.

In vitro competition assays. In vitro competition experiments were used to measure the relative fitness of the $m c r-1$ HLCRMs, E. coli TOP10 ( $m c r-1 / \mathrm{pBAD})$ and E. coli TOP10 (pBAD only) and E. coli TOP10. These strains were competed against a GFP-labelled E. coli DH5-alpha carrying plasmid pHT315-pAphA3'-gfp for constitutive expression ${ }^{52}$ and flow cytometry was used to measure changes in the cell titre of the two strains during competition. All competitions were carried out in M9 medium (Sigma-Aldrich, UK) with six replicates per strain/condition, as previously described with some modifications ${ }^{53}$. For E. coli TOP10 ( $\left.m c r-1 / \mathrm{pBAD}\right)$, mcr- 1 expression was induced in a controlled way by adding different concentrations of L-arabinose $(0,0.0002,0.002,0.02$ and $0.2 \%, \mathrm{w} / \mathrm{v})$.

The bacteria were cultured overnight in LB supplemented with the appropriate antibiotics $\left(2 \mathrm{mg} \mathrm{l}^{-1}\right.$ of colistin for HLCRMs, or $100 \mathrm{mg} \mathrm{l}^{-1}$ of ampicillin (Fisher Chemical) for E. coli TOP10 (mcr-1/pBAD), E. coli TOP10 (pBAD) and the GFPlabelled E. coli DH5-alpha. The overnight cultures were diluted 1:400 in M9 broth and mixed at 1:1 ratio with GFP-labelled cells. Before starting the competitions, the exact initial proportion of fluorescent/non-fluorescent cells was estimated using flow cytometry (for details see below). If the actual ratio was close to $1: 1$, we started the competition by shifting the mixtures to a shaking incubator $\left(37^{\circ} \mathrm{C}, 225\right.$ r.p.m. $)$. Otherwise, the preparation procedure was repeated.

After $22 \mathrm{~h}$ ( $6 \mathrm{~h}$ for HLCRMs), the competed bacteria were diluted 1:400 in M9 and analysed on a flow cytometer to estimate the resulting proportion of labelled vs unlabelled cells. Flow cytometry was performed on an Accuri C6 (Becton Dickenson, Biosciences, UK). The cell densities were adjusted to give $\sim 1000$ events per microlitre. During data acquisition, a lower cut off was set at 10,000 for FSC-H and at 8000 for SSC-H. For each competition, we ensured that the GFP-labelled strain can be well separated from non-fluorescent strains by comparing non-mixed controls (overlap is usually $<2 \%$ of the cells). Relative fitness was calculated using formula:

$$
\text { Relative_fitness }=\frac{\log _{2}\left(\frac{p_{1}}{p_{0} / n_{\text {dilution }}}\right)}{\log _{2}\left(\frac{1-p_{1}}{\left(1-p_{0}\right) / n_{\text {dilution }}}\right)},
$$

where $p_{0}$ is an initial proportion of an unlabelled stain, and $p_{1}$ is a final proportion of an unlabelled stain after competition. The $n_{\text {dilution }}$ is the factor, which reflects a fold difference in cell density at the beginning and at the end of the competition. For HLCRMs, we expressed the fitness of daughter strains relative to their parental strains (ie, $f_{\text {daughter }} / f_{\text {parental }}$ ) and followed the procedure of error propagation to account for the uncertainty of the two estimates:

$$
\mathrm{SE}=\sqrt{\left(\frac{\mathrm{SD}_{\text {daughter }}}{\bar{f}_{\text {daughter }}}\right)^{2}+\left(\frac{\mathrm{SD}_{\text {parental }}}{\bar{f}_{\text {parental }}}\right)^{2}}
$$

where $\bar{f}$ and SD are a mean estimate and its SD for each corresponding strain based on six replicates. Similarly, the relative fitness of the HLCRMs at different L-arabinose concentrations was represented as the relative fitness at no induction (no Larabinose). The differences in fitness were tested using non-parametric Mann-Whitney test, and the $p$ values were adjusted by Bonferroni method.

LPS isolation and macrophage stimulation. LPS was extracted using LPS extraction kit (iNtRON Biotechnology, UK), according to the manufacturers' instructions. To eliminate protein contamination, treatment with protease $\mathrm{K}$ was performed prior to the extraction steps. The protease $\mathrm{K}$ (AppliChem, Germany) $(20 \mathrm{mg} / \mathrm{ml})$ was added to the cell mixture and incubated at $56^{\circ} \mathrm{C}$ for $1 \mathrm{~h}$. The efficiency of the LPS preparation was determined by Limulus Amebocyte Lysate (LAL chromogenic Endotoxin Quantitation kit, ThermoFisher) to measure the LPS concentration, according to the manufacturers' instructions.

THP-1 human cells (American Type Culture Collection, ATCC TIB-202 ${ }^{\mathrm{TM}}$ ) were cultured with the medium (RPMI-1640 medium supplemented with $10 \%$ foetal calf serum, HEPES, L-glutamine, ampicillin and streptomycin) (SigmaAldrich) in a humidified cell culture incubator with $5 \% \mathrm{CO}_{2}$ at $37^{\circ} \mathrm{C}$. THP-1 cells were differentiated into macrophage cells by stimulation with phorbol myristate acetate (PMA). Cells were centrifuged and resuspended in fresh supplemented RPMI-1604 medium to a concentration of $8 \times 10^{5}$ cells per ml. Phorbol myristate acetate $\left(10 \mathrm{ng} \mathrm{ml}^{-1}\right)$ was added to diluted cells, and then aliquoted into 24 -well plate by adding $1 \mathrm{ml}$ of cell suspension per well. The differentiation of THP-1 monocytes were complete after $48 \mathrm{~h}$ of incubation, as exemplified by induction of adherent cells phenotype under the microscope.

Phorbol myristate acetate and any non-adherent cells were removed by replacement of the medium with fresh medium prior to addition of serial concentrations of LPS $(4.5,0.45,0.045,0.0045,0.00045 \mathrm{ng} / \mathrm{ml})$ to the appropriate wells in triplicate. Differentiated THP-1 cell cultures without LPS served as the negative controls. The wells are thoroughly mixed and incubated at $37^{\circ} \mathrm{C}$ for $24 \mathrm{~h}$. The samples were collected at $4,6,8$ and $24 \mathrm{~h}$ and stored at $-20^{\circ} \mathrm{C}$. The production of macrophage-derived cytokines (IL-6 and TNF-alpha) were analysed using DuoSet ${ }^{\circledR}$ ELISA kit (R\&D systems), and cytokine concentrations were calculated according to the manufacturers' instructions. 
Morphological analysis by TEM. Overnight cultures were diluted into $50 \mathrm{ml}$ of fresh media supplemented with $100 \mathrm{mg} \mathrm{l}^{-1}$ ampicillin for $m c r-1 / \mathrm{pBAD}$ and 2 $\mathrm{mg}^{-1}$ colistin for PN21 strains, respectively. For $m c r-1 / \mathrm{pBAD}$ strain, $0.2 \%$ of $\mathrm{L}$-arabinose was added to induce the overexpression of $m c r-1$. After $8 \mathrm{~h}$ incubation, samples were fixed by addition of glutaraldehyde to the broth to a final concentration of $1 \%$. Bacteria were harvested by collection onto $0.45 \mathrm{~mm}$ pore filters, gently scraped off and dispersed in $4 \%$ low melting point agarose at $50^{\circ} \mathrm{C}$. Preparations were allowed to gel at room temperature and cut into $1 \mathrm{~mm}$ cubes. Cubes were post-fixed for $2 \mathrm{~h}$ in $2 \%$ uranyl acetate, washed for $3 \times 20 \mathrm{~min}$ in reverse osmosis purified water and dehydrated through graded propan-2-ol (50\%, 70\%, $90 \%$ for $10 \mathrm{~min}$ each, $100 \%$ for $2 \times 15 \mathrm{~min}$ ), infiltrated with LR white acrylic resin (London Resin Company, Aldermaston, UK) (50\% in propan-2-ol 30 for min, neat resin for $4 \times 20 \mathrm{~min}$ ) placed in size 0 gelatine capsules with fresh resin and heat polymerised overnight at $50^{\circ} \mathrm{C}$. Thin $(80 \mathrm{~nm})$ sections were cut on an Ultracut E. ultramicrotome with a glass knife and collected onto 300 mesh copper grids, stained with lead citrate and examined in a Philips CM12 (FEI UK Ltd. UK) TEM at $80 \mathrm{kV}$. Digital images were captured with a Megaview III digital camera and AnalySIS (Soft Imaging System GmbH, Germany).

Galleria mellonella infection model. In vivo virulence of seven wild-type MCRPEC isolates and their HLCRMs (day 0, 3, 7, 11 and 14) were evaluated using G. mellonella infection model. The wax moth G.mellonella in larval stage (Live Foods UK Ltd., http://www.livefood.co.uk) were stored in dark and used within 3 days from shipment. Prior to inoculation into larvae, bacterial pellets were washed with sterile saline and then diluted to an appropriate cell density. Using a $50 \mu \mathrm{l} \mathrm{Hamilton}$ syringe, $10 \mu \mathrm{l}$ aliquots of serially diluted bacterial suspension (from $10^{3}$ to $10^{7}$ c.f.u. per ml) were injected into the haemocoel of each larvae, through the rear left pro-leg ${ }^{54-57}$. A group of 10 larvae were randomly chosen to inject for each level of inoculation in triplicate. Followed by injection, larvae were incubated at $37^{\circ} \mathrm{C}$, and the survival of larvae was monitored daily for 3 days. Death was denoted when larvae no longer responded to touch. Results were analysed by Kaplan-Meier survival curves (GraphPad Prism statistics software). For all experiments, three control groups were used: 10 larvae in first groups were injected with $10 \mu \mathrm{l}$ sterile saline, the second group included larvae that received mock injection to ensure death was not caused by physical trauma, and the larvae in third group with no injection. In all cases, no dead larvae were observed in the control groups.

S1 nuclease-based pulsed-field gel electrophoresis. To investigate whether $\mathrm{mcr}$ 1 genes were located on plasmids or chromosome, endonuclease S1 pulsed-field gel electrophoresis were assayed as previously described ${ }^{58}$. Briefly, bacterial DNA was prepared in agarose blocks and digested with 1 unit of S1 nuclease (Invitrogen). Electrophoresis was conducted on a CHEF-DR III apparatus (Bio-Rad, Hercules, CA, USA) under the following conditions: $6 \mathrm{~V} \mathrm{~cm}^{-1}$ at $14^{\circ} \mathrm{C}$, with an initial pulse time of $4 \mathrm{~s}$ and a final pulse time of $45 \mathrm{~s}$ for $18 \mathrm{~h}$. In-gel hybridisation was done with a $m c r-1$ probe labelled with ${ }^{32} \mathrm{P}$ (Stratgene, Amsterdam, Netherlands) with a random primer method according to manufacturers' instruction.

\section{Whole-genome sequencing and bioinformatics analysis. Total gDNA was} extracted from an overnight culture $(2 \mathrm{ml})$ on a QIAcube automated system (Qiagen). Following extraction, gDNA was quantified by fluorometric methods using a Qubit (ThermoFisher Scientific), with quality ratios of gDNA (A260/280 and 260/230) determined via Nanodrop (ThermoFisher Scientific). Genomic DNA libraries are prepared for whole-genome sequencing using the NexteraXT kit (Illumina), as described by the manufacturer. Paired end sequencing was performed using the Illumina MiSeq platform (MiSeq Reagent V3 Kit; $2 \times 300$ cycles). For each $E$. coli isolate, at least $80 \times$ coverage was generated. Raw sequence reads were trimmed using Trim Galore and the genomes were de novo-assembled into contigs using SPAdes (3.9.0) with pre-defined kmers set. Raw reads were also assembled with Geneious (10.0.9; Biomatters Ltd.) de novo assembler, set at medium sensitivity for analysis of paired Illumina reads. Geneious was used to map both sets of contigs to reference genes identified by closest BLAST homology and was also used to annotate genes from closest homologues in NCBI Genome database. Resistance genes were identified using Resfinder within CGE ${ }^{59}$, and wgMLST profiles were generated using the CGE platform coupled with the PubMLST.org database ${ }^{60}$. Plasmids were identified within the genome assembly and typed using Plasmidfinder ${ }^{61}$.

Analysis of lipid A modifications. A total of $200 \mathrm{ml}$ overnight culture were harvested by centrifugation at $10,000 \times g$ for $10 \mathrm{~min}$. Lipid A was extracted as previously described ${ }^{62}$. In brief, the pellets were washed twice and resuspended in $1 \times$ PBS, followed by adding single-phase Bligh-dyer solvent (chloroform: methanol: water; $1: 2: 0.8, \mathrm{v} / \mathrm{v} / \mathrm{v}$ ). The mixtures were incubated for $20 \mathrm{~min}$ at room temperature and centrifuged at $2000 \times g$ for $20 \mathrm{~min}$. The LPS pellets washed with the single-phase Bligh-Dyer solvent and resuspended in hydrolysis buffer by boiling the samples in $50 \mathrm{mM}$ sodium acetate ( $\mathrm{pH} 4.5)$. The samples were sonicated twice ( $20 \mathrm{~s}$ per burst) and incubated the samples in a boiling water bath for $30 \mathrm{~min}$. Then the lipid A was extracted using a two-phase Blign-Dyer solvent (chloroform:methanol: water; 2:2:1.8, v/v/v). Once extracted, $10 \mu \mathrm{l}$ of the purified Lipid A dissolved in chloroform-methonal $(2: 1, \mathrm{v} / \mathrm{v})$ were analysed using a ESI-QTOF mass spectrometery (Waters Synapt HR-MS) in the negative-ion mode. The spectral data were used to analyse the structures of bacterial lipid A from MCRPEC (E. coli
W3110 with plasmid pUC19-mcr-1) and non-MCRPEC (E. coli W3110 with empty vector $\mathrm{pUC19})^{9}$.

Data availability. Genomic sequences of bacterial strains have been deposited in the NCBI GenBank with accession codes MG489944, MG557851, MG557852, MG557853, and MG557854; there are two sequencing data still outstanding. Other relevant data supporting the findings of the study are available in this article and its Supplementary Information files, or from the corresponding authors upon request.

Received: 9 May 2017 Accepted: 9 November 2017

Published online: 12 December 2017

\section{References}

1. Jeannot, K., Bolard, A. \& Plésiat, P. Resistance to polymyxins in Gram-negative organisms. Int. J. Antimicrob. Agents 49, 526-535 (2017).

2. Olaitan, A. O. \& Li, J. Emergence of polymyxin resistance in Gram-negative bacteria. Int. J. Antimicrob. Agents 48, 581-582 (2016).

3. Olaitan, A. O., Morand, S. \& Rolain, J.-M. Mechanisms of polymyxin resistance: acquired and intrinsic resistance in bacteria. Front. Microbiol. 5, 643 (2014).

4. Wang, Y. et al. Comprehensive resistome analysis reveals the prevalence of NDM and MCR-1 in Chinese poultry production. Nat. Microbiol. 2, 16260 (2017).

5. Wang, J. et al. The role of wildlife (wild birds) in the global transmission of antimicrobial resistance genes. Zool. Res. 38, 55 (2017).

6. Unger, F. et al. Imported reptiles as a risk factor for the global distribution of Escherichia coli harbouring the colistin resistance gene mcr-1. Int. J. Antimicrob. Agents 49, 122-123 (2017).

7. Zurfuh, K. et al. Occurrence of the plasmid-borne mcr-1 colistin resistance gene in extended-spectrum- $\beta$-lactamase-producing Enterobacteriaceae in river water and imported vegetable samples in Switzerland. Antimicrob. Agents Chemother. 60, 2594-2595 (2016).

8. Sun, P. et al. Occurrence of blaKPC-2, blaCTX-M, and mcr-1 in Enterobacteriaceae from well water in rural China. Antimicrob. Agents Chemother. 61, e02569-02516 (2017).

9. Liu, Y.-Y. et al. Emergence of plasmid-mediated colistin resistance mechanism MCR-1 in animals and human beings in China: a microbiological and molecular biological study. Lancet Infect. Dis. 16, 161-168 (2016).

10. Wang, Y. et al. Prevalence, risk factors, outcomes, and molecular epidemiology of mcr-1-positive Enterobacteriaceae in patients and healthy adults from China: an epidemiological and clinical study. Lancet Infect. Dis. 17, 390-399 (2017).

11. Fernandes, M. R. et al. First report of the globally disseminated IncX4 plasmid carrying the $m c r-1$ gene in a colistin-resistant Escherichia coli sequence type 101 isolate from a human infection in Brazil. Antimicrob. Agents Chemother. 60, 6415-6417 (2016).

12. Hasman H., et al. Detection of mcr-1 encoding plasmid-mediated colistinresistant Escherichia coli isolates from human bloodstream infection and imported chicken meat, Denmark 2015. Euro Surveill. 20, 49 (2015).

13. Torpdahl, M. et al. Detection of mcr-1-encoding plasmid-mediated colistinresistant Salmonella isolates from human infection in Denmark. Int. J. Antimicrob. Agents 49, 261-262 (2017)

14. Coetzee, J. et al. Clinical alert-emergence of plasmid-mediated colistin resistance (MCR-1) among Escherichia coli isolated from South African patients: in practice. S. Afr. Med. J. 106, 449-450 (2016).

15. Quan, J. et al. Prevalence of mcr-1 in Escherichia coli and Klebsiella pneumoniae recovered from bloodstream infections in China: a multicentre longitudinal study. Lancet Infect. Dis. 17, 400-410 (2017)

16. He, Q.-w et al. Molecular characteristic of mcr-1 producing Escherichia coli in a Chinese university hospital. Ann. Clin. Microbiol. Antimicrob. 16, 32 (2017).

17. de la Tabla, V. O. et al. Detection of the high-risk clone ST131 of Escherichia coli carrying the colistin resistance gene mcr-1 and causing acute peritonitis. Int. J. Antimicrob. Agents 49, 115-116 (2017).

18. Gao, R. et al. Dissemination and mechanism for the MCR-1 colistin resistance. PLoS Pathog. 12, e1005957 (2016).

19. Hinchliffe, P. et al. Insights into the mechanistic basis of plasmid-mediated colistin resistance from crystal structures of the catalytic domain of MCR-1. Sci. Rep. 7, 39392 (2017).

20. Dénervaud Tendon, V., Poirel, L. \& Nordmann, P. Transferability of the mcr-1 colistin resistance gene. Microb. Drug Resist. 23, 813-814 (2017).

21. Bontron, S., Poirel, L. \& Nordmann, P. Real-time PCR for detection of plasmidmediated polymyxin resistance (mcr-1) from cultured bacteria and stools. $J$. Antimicrob. Chemother. 71, 2318-2320 (2016).

22. Pristovsek, P. \& Kidric, J. Solution structure of polymyxins B and E and effect of binding to lipopolysaccharide: an NMR and molecular modeling study. J. Med. Chem. 42, 4604-4613 (1999).

23. Park, B. S. et al. The structural basis of lipopolysaccharide recognition by the TLR4-MD-2 complex. Nature 458, 1191-1195 (2009). 
24. Jønsson R., Struve C., Jenssen H. \& Krogfelt K. A. The wax moth Galleria mellonella as a novel model system to study enteroaggregative Escherichia coli pathogenesis. Virulence 0, 1-6 (2016).

25. Champion, O. L., Wagley, S. \& Titball, R. W. Galleria mellonella as a model host for microbiological and toxin research. Virulence 7, 840-845 (2016).

26. Andersson, D. I. \& Levin, B. R. The biological cost of antibiotic resistance. Curr. Opin. Microbiol. 2, 489-493 (1999).

27. Vogwill, T. \& MacLean, R. C. The genetic basis of the fitness costs of antimicrobial resistance: a meta-analysis approach. Evolut. Appl. 8, 284-295 (2015).

28. Andersson, D. I. \& Hughes, D. Antibiotic resistance and its cost: is it possible to reverse resistance? Nat. Rev. Microbiol. 8, 260-271 (2010).

29. MacLean, R. C., Hall, A. R., Perron, G. G. \& Buckling, A. The population genetics of antibiotic resistance: integrating molecular mechanisms and treatment contexts. Nat. Rev. Genet. 11, 405-414 (2010).

30. Guillard, T., Pons, S., Roux, D., Pier, G. B. \& Skurnik, D. Antibiotic resistance and virulence: understanding the link and its consequences for prophylaxis and therapy. Bioessays 38, 682-693 (2016).

31. Roux, D. et al. Fitness cost of antibiotic susceptibility during bacterial infection. Sci. Transl. Med. 7, 297ra114 (2015).

32. Schroeder, M., Brooks, B. D. \& Brooks, A. E. The complex relationship between virulence and antibiotic resistance. Genes 8, 39 (2017).

33. Kumarasamy, K. K. et al. Emergence of a new antibiotic resistance mechanism in India, Pakistan, and the UK: a molecular, biological, and epidemiological study. Lancet Infect. Dis. 10, 597-602 (2010).

34. Coyne, S., Courvalin, P. \& Perichon, B. Efflux-mediated antibiotic resistance in Acinetobacter spp. Antimicrob. Agents Chemother. 55, 947-953 (2011).

35. Eckert, B. \& Beck, C. F. Overproduction of transposon Tn10-encoded tetracycline resistance protein results in cell death and loss of membrane potential. J. Bacteriol. 171, 3557-3559 (1989).

36. Moyed, H. S., Nguyen, T. T. \& Bertrand, K. P. Multicopy Tn10 tet plasmids confer sensitivity to induction of tet gene expression. J. Bacteriol. 155, 549-556 (1983).

37. Walsh, T. R. \& Wu, Y. China bans colistin as a feed additive for animals. Lancet Infect. Dis. 16, 1102 (2016).

38. Comas, I. et al. Whole-genome sequencing of rifampicin-resistant Mycobacterium tuberculosis strains identifies compensatory mutations in RNA polymerase genes. Nat. Genet. 44, 106-110 (2012).

39. Needham, B. D. \& Trent, M. S. Fortifying the barrier: the impact of lipid A remodelling on bacterial pathogenesis. Nat. Rev. Microbiol. 11, 467-481 (2013).

40. Matsuura, M. Structural modifications of bacterial lipopolysaccharide that facilitate Gram-negative bacteria evasion of host innate immunity. Front. Immunol. 4, 109 (2013).

41. Tamayo, R. et al. Identification of cptA, a PmrA-regulated locus required for phosphoethanolamine modification of the Salmonella enterica serovar typhimurium lipopolysaccharide core. J. Bacteriol. 187, 3391-3399 (2005).

42. Trombley, M. P. et al. Phosphoethanolamine transferase LptA in haemophilus ducreyi modifies lipid A and contributes to human defensin resistance in vitro. PLoS ONE 10, e0124373 (2015)

43. John, C. M. et al. Lipooligosaccharide structures of invasive and carrier isolates of Neisseria meningitidis are correlated with pathogenicity and carriage. J. Biol. Chem. 291, 3224-3238 (2016).

44. Kidd, T. J. et al. A Klebsiella pneumoniae antibiotic resistance mechanism that subdues host defences and promotes virulence. EMBO Mol. Med. 9, e201607336 (2017).

45. Cullen, T. W. et al. EptC of Campylobacter jejuni mediates phenotypes involved in host interactions and virulence. Infect. Immun. 81, 430-440 (2013).

46. Zughaier, S. M., Kandler, J. L., Balthazar, J. T. \& Shafer, W. M. Phosphoethanolamine modification of Neisseria gonorrhoeae lipid A reduces autophagy flux in macrophages. PLoS ONE 10, e0144347 (2015).

47. MacLean, R. C. \& Vogwill, T. Limits to compensatory adaptation and the persistence of antibiotic resistance in pathogenic bacteria. Evol. Med. Public Health 2015, 4-12 (2015).

48. Quesada, A. et al. Polymorphism of genes encoding PmrAB in colistin-resistant strains of Escherichia coli and Salmonella enterica isolated from poultry and swine. J. Antimicrob. Chemother. 70, 71-74 (2015).

49. Sun, S., Negrea, A., Rhen, M. \& Andersson, D. I. Genetic analysis of colistin resistance in Salmonella enterica serovar typhimurium. Antimicrob. Agents Chemother. 53, 2298-2305 (2009).

50. Guzman, L. M., Belin, D., Carson, M. J. \& Beckwith, J. Tight regulation, modulation, and high-level expression by vectors containing the arabinose PBAD promoter. J. Bacteriol. 177, 4121-4130 (1995).

51. Heydorn, A. et al. Quantification of biofilm structures by the novel computer program COMSTAT. Microbiology 146, 2395-2407 (2000).

52. Daou, N. et al. IlsA, a unique surface protein of Bacillus cereus required for iron acquisition from heme, hemoglobin and ferritin. PLoS Pathog. 5, e1000675 (2009).

53. San Millan, A., Escudero, J. A., Gifford, D. R., Mazel, D. \& MacLean, R. C. Multicopy plasmids potentiate the evolution of antibiotic resistance in bacteria. Nat. Ecol. Evol. 1, 0010 (2016).
54. Peleg, A. Y. et al. Galleria mellonella as a model system to study Acinetobacter baumannii pathogenesis and therapeutics. Antimicrob. Agents Chemother. 53, 2605-2609 (2009).

55. Williamson, D. A., Mills, G., Johnson, J. R., Porter, S. \& Wiles, S. In vivo correlates of molecularly inferred virulence among extraintestinal pathogenic Escherichia coli (ExPEC) in the wax moth Galleria mellonella model system. Virulence 5, 388-393 (2014).

56. Brennan, M., Thomas, D. Y., Whiteway, M. \& Kavanagh, K. Correlation between virulence of Candida albicans mutants in mice and Galleria mellonella larvae. FEMS Immunol. Med. Microbiol. 34, 153-157 (2002).

57. Seed, K. D. \& Dennis, J. J. Development of Galleria mellonella as an alternative infection model for the Burkholderia cepacia complex. Infect. Immun. 76, 1267-1275 (2008).

58. Yong, D. et al. Characterization of a new metallo-beta-lactamase gene, bla (NDM-1), and a novel erythromycin esterase gene carried on a unique genetic structure in Klebsiella pneumoniae sequence type 14 from India. Antimicrob. Agents Chemother. 53, 5046-5054 (2009).

59. Zankari, E. et al. Identification of acquired antimicrobial resistance genes. $J$. Antimicrob. Chemother. 67, 2640-2644 (2012).

60. Larsen, M. V. et al. Multilocus sequence typing of total-genome-sequenced bacteria. J. Clin. Microbiol. 50, 1355-1361 (2012)

61. Carattoli, A. et al. In silico detection and typing of plasmids using PlasmidFinder and plasmid multilocus sequence typing. Antimicrob. Agents Chemother. 58, 3895-3903 (2014).

62. Hankins, J. V., Madsen, J. A., Needham, B. D., Brodbelt, J. S. \& Trent, M. S. The outer membrane of Gram-negative bacteria: lipid A isolation and characterization. Methods Mol. Biol. 966, 239-258 (2013)

\section{Acknowledgements}

We thank Dr Jonathan Tyrell (Division of Infection and Immunity, Cardiff University) for useful suggestions on Galleria infection model, Dr M. Toleman (Division of Infection and Immunity, Cardiff University) for his valuable advice on genome sequencing, and Professor W. Jiang (Cardiff China Medical Research Collaborative, Cardiff University) for access to qPCR machine (Applied Biosystems ${ }^{\text {TM }}$ StepOnePlus ${ }^{\circledR}, \mathrm{UK}$ ). This work was supported by MRC grant DETER-XDR-CHINA (MR/P007295/1). Q.Y. is funded by a CSC Scholarship. D.O.A. benefits a Geneva University Hospitals (HUG) and Swiss National Science Foundation (P300PB_171601) overseas fellowship. P.N. and U.T. are funded by Royal Golden Jubilee-PhD Program from Thailand Research Fund and Rajamangala University of Technology Lanna (PHD/0054/2555).

\section{Author contributions}

Conceived and designed the experiment: T.W., J.S., O.B.S. and Ji.S. Performed the experiments: Q.Y., M.L., O.B.S., D.O.A., K.S., E.P., L.P., M.P., P.N. and U.T. Analysed the data: Q.Y., O.B.S., D.O.A., P.H., A.P., Y.W., S.W., Y.S., C.M. and D.T. Writing of the main paper: T.W. and Q.Y. All authors contributed extensively to the work presented in this paper. All authors discussed the results and implications and commented on the manuscript at all stages.

\section{Additional information}

Supplementary Information accompanies this paper at https://doi.org/10.1038/s41467017-02149-0.

Competing interests: The authors declare no competing financial interests.

Reprints and permission information is available online at http://npg.nature.com/ reprintsandpermissions/

Publisher's note: Springer Nature remains neutral with regard to jurisdictional claims in published maps and institutional affiliations.

\footnotetext{
Open Access This article is licensed under a Creative Commons Attribution 4.0 International License, which permits use, sharing adaptation, distribution and reproduction in any medium or format, as long as you give appropriate credit to the original author(s) and the source, provide a link to the Creative Commons license, and indicate if changes were made. The images or other third party material in this article are included in the article's Creative Commons license, unless indicated otherwise in a credit line to the material. If material is not included in the article's Creative Commons license and your intended use is not permitted by statutory regulation or exceeds the permitted use, you will need to obtain permission directly from the copyright holder. To view a copy of this license, visit http://creativecommons.org/ licenses/by/4.0/.
}

(c) The Author(s) 2017 